\title{
On Ve-Degree and Ev-Degree Topological Properties of Hyaluronic Acid-Anticancer Drug Conjugates with QSPR
}

\author{
Syed Ajaz K. Kirmani, ${ }^{1}$ Parvez Ali $\mathbb{D}^{2},{ }^{2}$ Faizul Azam $\mathbb{D D}^{3}$ and Parvez Ahmad Alvi ${ }^{4}$ \\ ${ }^{1}$ Department of Electrical Engineering, College of Engineering, Qassim University, Unaizah, Saudi Arabia \\ ${ }^{2}$ Department of Mechanical Engineering, College of Engineering, Qassim University, Unaizah, Saudi Arabia \\ ${ }^{3}$ Department of Pharmaceutical Chemistry \& Pharmacognosy, Unaizah College of Pharmacy, Qassim University, \\ Unaizah, Saudi Arabia \\ ${ }^{4}$ Department of Physics, Banasthali Vidiapith, Vanasthali 304022, Rajasthan, India
}

Correspondence should be addressed to Parvez Ali; parvezamu1@gmail.com

Received 9 April 2021; Accepted 14 June 2021; Published 3 July 2021

Academic Editor: Maria N. D.S. Cordeiro

Copyright (C) 2021 Syed Ajaz K. Kirmani et al. This is an open access article distributed under the Creative Commons Attribution License, which permits unrestricted use, distribution, and reproduction in any medium, provided the original work is properly cited.

\begin{abstract}
The design of the quantitative structure-property/activity relationships for drug-related compounds using theoretical methods relies on appropriate molecular structure representations. The molecular structure of a compound comprises all the information required to determine its chemical, biological, and physical properties. These properties can be assessed by employing a graph theoretical descriptor tool widely known as topological indices. Generalization of descriptors may reduce not only the number of molecular graph-based descriptors but also improve existing results and provide a better correlation to several molecular properties. Recently introduced ve-degree and ev-degree topological indices have been successfully employed for development of models for the prediction of various biological activities/properties. In this article, we propose the general ve-inverse sum indeg index $\operatorname{ISI}_{(\alpha, \beta)}^{\mathrm{ve}}(G)$ and general ve-Zagreb index $M_{\alpha}^{\mathrm{ve}}(G)$ of graph $G$ and compute $\operatorname{ISI}_{(\alpha, \beta)}^{\mathrm{ve}}(G), M_{\alpha}^{\mathrm{ve}}(G)$, and $M_{\alpha}^{\mathrm{ev}}(G)$ (general evdegree index) of hyaluronic acid-curcumin/paclitaxel conjugates, renowned for its potential anti-inflammatory, antioxidant, and anticancer properties, by using molecular structure analysis and edge partitioning technique. Several ve-degree- and ev-degreebased topological indices are obtained as a special case of $\operatorname{ISI}_{(\alpha, \beta)}^{\mathrm{ve}}(G), M_{\alpha}^{\mathrm{ve}}(G)$, and $M_{\alpha}^{\mathrm{ev}}(G)$. Furthermore, QSPR analysis of $\operatorname{ISI}_{(\alpha, \beta)}^{\mathrm{ve}}(G), M_{\alpha}^{\mathrm{ve}}(G)$, and $M_{\alpha}^{\mathrm{ev}}(G)$ for particular values of $\alpha$ and $\beta$ is performed, which reveals their predicting power. These results allow researchers to better understand the physicochemical properties and pharmacological characteristics of these conjugates.
\end{abstract}

\section{Introduction}

In this period of exponential technological development, pharmaceutical and chemical technologies have grown rapidly. A large number of new drugs, nanomaterials, and crystalline materials are therefore being produced each year. A substantial amount of work is required to establish the pharmacological, chemical, and biological characteristics of drugs with an increase in the development of medicines. A large number of chemical experiments are needed to determine the pharmacological, chemical, and biological properties of these new compounds and drugs, which significantly increases the workload of pharmaceutical and chemical researchers. These properties of the drugs may be predicted without using any weight lab by analyzing the molecular structure of the relevant drug using a well-known tool of chemical graph theory known as topological index.

Topological indices are simply defined as numerical values associated with chemical constitution, which is used for correlation of chemical structure with numerous characteristics such as chemical reactivity, pharmacological activity, and physical properties. Topological indices have been used to explain and improve the statistical features of drugs. Topological indices play a vital role in quantitative structureproperty relationship (QSAR) and the quantitative structure-activity relationship (QSPR) in predicting different physicochemical properties and bioactivity that contribute in the discovery of drugs $[1,2]$. 
In order to calculate topological indices, the structure of the drugs is represented as a graph known as molecular graphs, where each vertex indicates an atom and each edge represents a chemical bond between the atoms. Let $G=$ $(V, E)$ be a molecular graph with vertex set $V(G)$ and edge set $E(G)$. We denote the number of vertices and edges in a graph $G$ by $|V(G)|$ and $|E(G)|$, respectively. The degree of vertex $u \in V(G)$ is denoted by $\operatorname{deg}(u)$ or $d(u)$ and is the number of vertices that are adjacent to $u$. The edge connecting the vertices $u$ and $v$ is denoted by $e=u v$, where $e \in E(G)$. The set of all vertices which is adjacent to $u$ is called the open neighborhood of $u$ and denoted by $N(u)$. If we add the vertex $u$ to $N(u)$, then we get the closed neighborhood of $u, N[u]$.

The concept of the topological index was introduced by Wiener [3] while working on the boiling points of alkanes. Topological indices are categorized in a variety of groups, such as degree-based, distance-based, and counting-based [4]. Among them, topological indexes based on degrees play a significant role in theoretical chemistry and pharmacology. The most widely used topological indices in chemical and mathematical literature are the Randić index, Zagreb index, harmonic index, and Wiener index [4-12].

Degree-based topological indices have been studied extensively to test the properties of compounds and drugs as it is useful to make up the medicinal and chemical experimental defects. We encourage reader to refer [13-18] for more on topological indices of various drugs. Degree-based topological indices are widely used descriptors in QSAR/QSPR modeling due to their easy understandability, applicability, ease of computation, and their derivation without any experimental effort. QSPRs were developed recently for a set of seventeen anticancer drugs from amathaspiramide-E to tambjamine-K by using a set of thirteen degree-based topological indices [19]. Recently, Sarkar et al. [20] developed a QSAR model to predict the DNA-binding constant and growth-inhibiting concentration of twenty-three anthracycline drugs by using first Zagreb index, second Zagreb index, and several others topological indices. For several other examples of degree-based topological indices used in QSAR/QSPR models, we refer $[1,21,22]$.

In recent past, Chellali et al. [23] defined two novel degree concepts, ev-degrees and ve-degrees, and explored some basic mathematical properties of both novel graph invariants with regard to graph regularity and irregularity. The ve-degree of the vertex $u$, denoted by $\operatorname{deg}_{\mathrm{ve}}(u)$ or $d_{\mathrm{ve}}(u)$, equals the number of different edges that is incident to any vertex from the closed neighborhood of $u, N[u]$. The evdegree of the edge $e$, denoted by $\operatorname{deg}_{\mathrm{ev}}(e)$ or $d_{\mathrm{ev}}(e)$, equals the number of vertices of the union of the closed neighborhoods of $u$ and $v$. Clearly, $d_{\mathrm{ev}}(e)=d(u)+d(v)-n_{e}$, where $n_{e}$ is the number of triangles in which the edge $e$ lies in.

It was recommended that the chemical applicability of the total ve-degree $\left(\sum_{v \in V(G)} d_{\mathrm{ve}}(v)\right)$ and the total ev-degree $\left(\sum_{e \in E(G)} d_{\mathrm{ev}}(e)\right)$ could be an interesting problem in view of chemistry and chemical graph theory. In the light of this suggestion, Ediz [24] introduced the ev-degree Zagreb index of the graph $G M^{\mathrm{ev}}(G)=\sum_{e \in E(G)} d_{\mathrm{ev}}(e)^{2}$, the first ve-degree Zagreb alpha index of the graph $G$ $M_{1}^{\alpha \mathrm{ve}}(G)=\sum_{v \in V(G)} d_{\mathrm{ve}}(v)^{2}$, the first ve-degree Zagreb beta index of the graph $G M_{1}^{\beta \mathrm{ve}}(G)=\sum_{u v \in E(G)}\left(d_{\mathrm{ve}}(u)+d_{\mathrm{ve}}(v)\right)$, the second ve-degree Zagreb index of the graph $G$ $M_{2}^{\mathrm{ve}}(G)=\sum_{u v \in E(G)} d_{\mathrm{ve}}(u) d_{\mathrm{ve}}(v)$, and the ve-degree Randic index of the graph $G R^{\mathrm{ve}}(G)=\sum_{u v \in E(G)}\left(d_{\mathrm{ve}}(u) d_{\mathrm{ve}}(v)\right)^{-(1 / 2)}$ and compared these newly defined indices with the other well-known, most widely used topological indices by modeling some physicochemical properties of octane isomers. It has been shown that the ev-degree Zagreb index, the ve-degree Zagreb index, and the ve-degree Randić indices have a better correlation than the Wiener, Zagreb, and Randić indices for predicting certain basic physicochemical properties of octanes. Later, Ediz defined the ev-degree Randić index as $R^{\mathrm{ev}}(G)=\sum_{e \in E(G)} d_{\mathrm{ev}}(e)^{-(1 / 2)}$ and show that it gives a better correlation than the Randić index to predict the entropy, acentric factor, and standard enthalpy of vaporization of octanes [25]. Sahin and Ediz [26] have shown that ev-degree and ve-degree Narumi-Katayama indices can be used as potential tools for QSPR analysis. Ediz [27] defined ve-degree atom-bond connectivity, ve-degree geometric-arithmetic, ve-degree harmonic, and ve-degree sum-connectivity indices as parallel to their corresponding classical degree versions as

$$
\begin{aligned}
A B C^{\mathrm{ve}}(G) & =\sum_{u v \in E(G)} \sqrt{\frac{d_{\mathrm{ve}}(u)+d_{\mathrm{ve}}(v)-2}{d_{\mathrm{ve}}(u) d_{\mathrm{ve}}(v)}}, \\
\mathrm{GA}^{\mathrm{ve}}(G) & =\sum_{u v \in E(G)} \frac{2 \sqrt{d_{\mathrm{ve}}(u) d_{\mathrm{ve}}(v)}}{d_{\mathrm{ve}}(u)+d_{\mathrm{ve}}(v)} \\
H^{\mathrm{ve}}(G) & =\sum_{u v \in E(G)} \frac{2}{d_{\mathrm{ve}}(u)+d_{\mathrm{ve}}(v)}, \\
\chi^{\mathrm{ve}}(G) & =\sum_{u v \in E(G)}\left(d_{\mathrm{ve}}(u)+d_{\mathrm{ve}}(v)\right)^{-1 / 2} .
\end{aligned}
$$

It is shown that the ve-degree sum-connectivity index gives a better correlation than Wiener, Zagreb, and Randić indices to predict the acentric factor of octanes. Horoldagva et al. [28] have explored some mathematical aspects of vedegree and ev-degree of a graph and have shown that there exists a highly ve-irregular graph of order $n$ for every positive integer $n(\neq 3,5)$.

Kulli [29] defined first and second hyper ve-degree as follows:

$$
\begin{aligned}
& \operatorname{HM}_{1}^{\mathrm{ve}}(G)=\sum_{u v \in E(G)}\left(d_{\mathrm{ve}}(u)+d_{\mathrm{ve}}(v)\right)^{2}, \\
& \operatorname{HM}_{2}^{\mathrm{ve}}(G)=\sum_{u v \in E(G)}\left(d_{\mathrm{ve}}(u) d_{(v e]}(v)\right)^{2} .
\end{aligned}
$$

Later, Kulli also introduced $F$-ve-degree, $F_{1}$-ve-degree indices, and arithmetic-geometric ve-degree indices [30-32] as 


$$
\begin{aligned}
F^{\mathrm{ve}}(G) & =\sum_{u v \in E(G)}\left[d_{\mathrm{ve}}(u)^{2}+d_{\mathrm{ve}}(v)^{2}\right], \\
F_{1}^{\mathrm{ve}}(G) & =\sum_{u \in V(G)} d_{\mathrm{ve}}(u)^{3}, \\
\mathrm{AG}^{\mathrm{ve}}(G) & =\sum_{u v \in E(G)} \frac{d_{\mathrm{ve}}(u)+d_{\mathrm{ve}}(v)}{2 \sqrt{d_{\mathrm{ve}}(u) d_{\mathrm{ve}}(v)}} .
\end{aligned}
$$

Very recently, Kulli [33] defined following ev-degree topological indices.

The modified ev-degree Zagreb index is

$$
\mathrm{mM}^{\mathrm{ev}}(G)=\sum_{e \in E(G)} \frac{1}{d_{\mathrm{ev}}(e)^{2}} .
$$

The ev-degree inverse index is

$$
\mathrm{ID}^{\mathrm{ev}}(G)==\sum_{e \in E(G)} \frac{1}{d_{\mathrm{ev}}(e)} .
$$

The F-ev-degree index is

$$
F^{\mathrm{ev}}(G)=\sum_{e \in E(G)} d_{\mathrm{ev}}(e)^{3} .
$$

The reciprocal ev-degree Randic index is

$$
\operatorname{RR}^{\mathrm{ev}}(G)=\sum_{e \in E(G)} d_{\mathrm{ev}}(e)^{1 / 2} .
$$

The following ve-degree topological indices are defined parallel to their corresponding classical degree versions [34-37] as follows:

Redefined third ve-degree Zagreb index is

$$
\operatorname{ReZG}_{3}^{\mathrm{ve}}(G)=\sum_{u v \in E(G)} d_{\mathrm{ve}}(u) d_{\mathrm{ve}}(v)\left(d_{\mathrm{ve}}(u)+d_{\mathrm{ve}}(v)\right) .
$$

Ve-degree inverse sum indeg index is

$$
\operatorname{ISI}^{\mathrm{ve}}(G)=\sum_{u v \in E(G)} \frac{d_{\mathrm{ve}}(u) d_{\mathrm{ve}}(v)}{d_{\mathrm{ve}}(u)+d_{\mathrm{ve}}(v)} .
$$

Inverse ve-degree index is

$$
\operatorname{ID}^{\mathrm{ve}}(G)=\sum_{u \in V(G)} \frac{1}{d_{\mathrm{ve}}(u)} .
$$

Zeroth order ve-degree index is

$$
\mathrm{ZD}^{\mathrm{ve}}(G)=\sum_{u \in V(G)} \frac{1}{\sqrt{d_{\mathrm{ve}}(u)}} .
$$

Modified first ve-degree index is

$$
\mathrm{mM}_{1}^{\mathrm{ve}}(G)=\sum_{u \in V(G)} \frac{1}{d_{\mathrm{ve}}(u)^{2}} .
$$

Various ve-degree and ev-degree topological indices for some silicate oxygen networks such as the dominating oxide network (DOX), regular triangulene oxide network (RTOX), and dominating silicate network (DSL) are considered in literature [27, 29-32]. Cancan investigated the Tickysim spiking neural network via ev-degree and ve-degree topological properties calculations giving information about the underlying topology of the Tickysim spiking neural network [38]. The ev-degree and ve-degree topological indices for Sierpinski gasket fractal are evaluated by Yamaç and Cancan [39]. Cancan et al. studied ve-degree Zagreb and Randić indices, ve-degree atom-bond connectivity, sum-connectivity, geometric-arithmetic, and harmonic topological properties of copper oxide [40,41]. Very recently, Chen et al. [42] investigated many topological properties of Cuprite. Cai et al. [43] computed various ve-degree and ev-degree topological indices for silicon carbide $\mathrm{Si}_{2} \mathrm{C}_{3}$-II $[p, q]$.

Recently, ev-degree- and ve-degree-based properties have been investigated for many anticancer drugs. Various ev-degree and ve-degree topological indices for the Doxloaded micelle comprising PEGPAsp block copolymer bioconjugate molecular structure have been investigated to predict some of its physicochemical properties by Rauf et al. [44]. A number of ve-degree and ev-degree topological indices for some newly defined thioTEPA-based anticancer drugs and alkylating agents based on the dual-target anticancer drug candidates have been investigated by Ediz et al. $[45,46]$.

Paclitaxel, a tricyclic diterpenoid compound having molecular formula $\mathrm{C}_{47} \mathrm{H}_{51} \mathrm{NO}_{14}$, is isolated from the bark of Taxus brevifolia. Its unique antiproliferative mechanism makes it an efficient anticancer drug [47]. It is an important medication that is prescribed in various forms of cancer in spite of its limitations, such as low solubility and relevant adverse effects. Curcumin, another natural compound of pharmaceutical importance is obtained from Curcuma longa, has been found to possess a wide range of pharmacological effects such as anti-inflammatory, antioxidant, antiproliferative, chemosensitizing, and cell cycle arrest [48]. In particular, it is recognized as a chemopreventive agent and used against cancer prevention and therapy [49]. Unfortunately, the poor bioavailability of curcumin in biological systems due to its low aqueous-solubility may jeopardize its usage in clinical practice [50].

However, water solubility and subsequent bioavailability of several compounds of therapeutic interests can be improved upon combining with carriers such as liposomes, polymeric micelles, nanospheres, emulsions, and polymers [51-53]. Natural polymers with an intrinsic cell-specific binding capability have a tremendous potential as a targetoriented drug carrier. For example, hyaluronic acid (HA), a polymer of naturally formed glycosaminoglycan polysaccharides consisting of $\beta-1,4-\mathrm{D}$-glucuronic acid and $\beta-1,3-\mathrm{N}$ acetylglucosamine units, has an appreciable affinity with cell-specific surface markers such as a cluster of differentiation 44 (CD44) and receptor for HA-mediated motility (RHAMM) [54]. HA and its derivatives are widely used as targeted drug delivery tools for a broad range of medicinal compounds [55]. Presence of three functional groups of carboxyl, amino, and acetyl amino groups on the main chain of hyaluronic acid offers valuable sites for chemical modification. Therefore, different antitumor drugs can be covalently bonded to HA, forming HA-drug conjugates. 
Galer et al. synthesized the HA-paclitaxel conjugate (HA-PTX), in order to reduce the toxicity of taxanes and improve the antitumor activity. It has been reported that curcumin conjugation with HA increases the solubility in water as well as the stability of curcumin at physiological $\mathrm{pH}$. In addition, curcumin conjugates with $\mathrm{HA}$ are considered to be a promising medicinal strategy for prolonging the release of curcumin at the target site, optimizing tissue distribution, and enhancing therapeutic outcomes [56]. Along with this, it has received considerable attention for not only increasing bioavailability but also for targeting tumor cells and tumor metastases for the treatment of various types of cancers $[57,58]$.

Recently, Buragohain et al. [59] proposed the general inverse sum indeg index, denoted by $\operatorname{ISI}_{(\alpha, \beta)}(G)$ and defined as

$$
\operatorname{ISI}_{(\alpha, \beta)}(G)=\sum_{u v \in E(G)}[d(u) d(v)]^{\alpha}[d(u)+d(v)]^{\beta} .
$$

Owing to enormous pharmaceutical interests of HAcurcumin conjugates, very recently, Ali et al. [60] investigated many degrees based topological indices and polynomial of HA-curcumin conjugates using the general inverse sum indeg index $\operatorname{ISI}_{(\alpha, \beta)}$.

Since the ve-degree index has been shown to have greater predictive ability, the current research on HA conjugates is being extended and seeks to investigate ve-degree and evdegree-based topological indices of the molecular structure of HA-curcumin conjugate and HA-paclitaxel conjugate as shown in Figures 1 and 2. This motivates us to define the general ve-inverse sum indeg index $\operatorname{ISI}_{(\alpha, \beta)}^{\mathrm{ve}}(G)$ as

$$
\operatorname{ISI}_{(\alpha, \beta)}^{\mathrm{ve}}(G)=\sum_{u v \in E(G)}\left[d_{\mathrm{ve}}(u) d_{\mathrm{ve}}(v)\right]^{\alpha}\left[d_{\mathrm{ve}}(u)+d_{\mathrm{ve}}(v)\right]^{\beta},
$$

where $\alpha$ and $\beta$ are some real numbers.

Table 1 enlists some of the ve-degree-based indices of graph $G$ that can be obtained from the generalized $\operatorname{ISI}_{(\alpha, \beta)}^{\mathrm{ve}}(G)$ index by only giving specific values to the parameters $\alpha$ and $\beta$.

Next, we define the general ve-Zagreb index as

$$
M_{\alpha}^{\mathrm{ve}}(G)=\sum_{u \in V(G)}\left[d_{\mathrm{ve}}(u)\right]^{\alpha},
$$

where $\alpha$ is some real number. In Table 2, some of the vedegree-based indices of graph $G$ that can be obtained from the general ve-Zagreb index $M_{\alpha}^{\mathrm{ve}}(G)$ by assigning particular values of the parameters are summarized.

Recently, Kulli [33] introduced the general ev-degree index of graph $G$ defined as

$$
M_{\alpha}^{\mathrm{ev}}(G)=\sum_{e \in E(G)}\left[d_{\mathrm{ev}}(e)\right]^{\alpha},
$$

where $\alpha$ is some real number. Table 3 summarizes ve-degreebased indices of graph $G$ that can be derived from the general ev-degree index of graph $G$ by giving certain values to parameters $\alpha$.

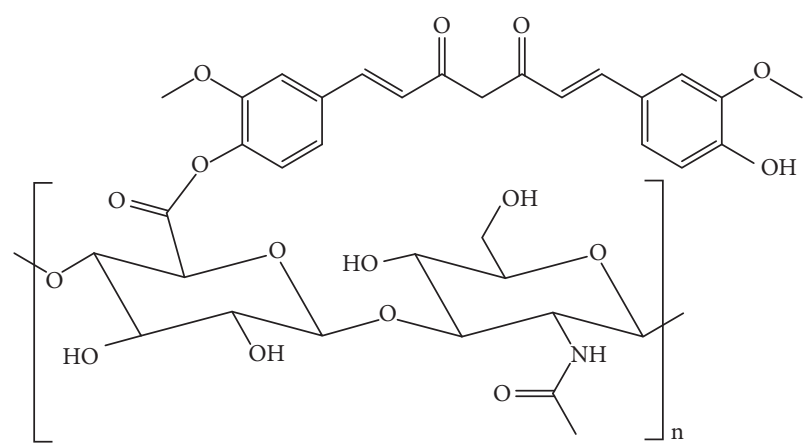

FIgURE 1: The molecular structure of hyaluronic acid-curcumin conjugates.

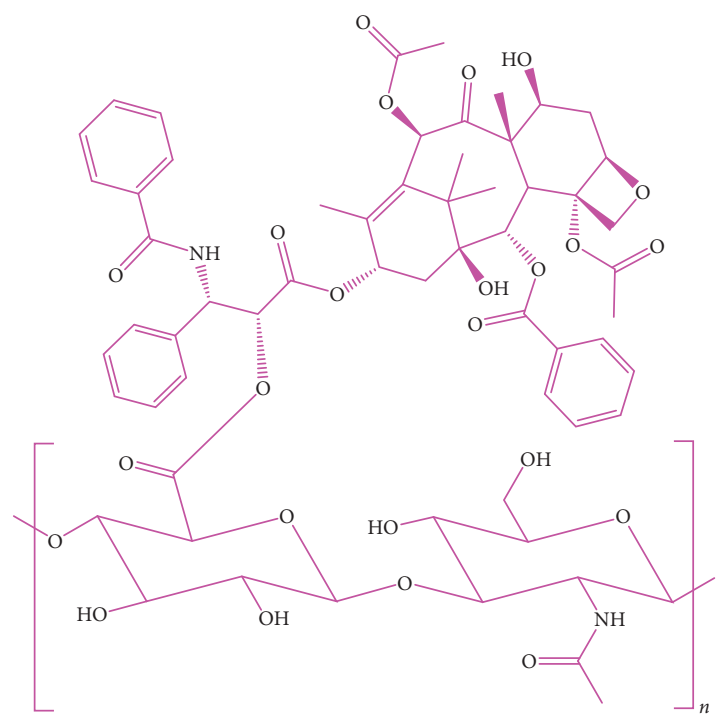

FIgURE 2: The molecular structure of hyaluronic acid-paclitaxel conjugates.

\section{Methodology and Main Results}

In order to obtain the results, we apply combinatorial computation, edge partitioning, vertex partitioning, and analytical techniques. In addition, we make use of ChemSketch for plotting the molecular graphs.

The following notation will be used in the discussion hereafter.

$$
\begin{aligned}
E_{i, j} & =\left\{u v \in E\left(G_{n}\right) \mid d_{\mathrm{ve}}(u)=i, d_{\mathrm{ve}}(v)=j\right\}, \\
V_{i} & =\left\{u \in V\left(G_{n}\right) \mid d_{\mathrm{ve}}(u)=i\right\}, \\
E_{i} & =\left\{e=u v \in E\left(G_{n}\right) \mid d_{\mathrm{ev}}(e)=i\right\}, \quad \text { where } G_{n}=\text { HAC or HAP. }
\end{aligned}
$$

2.1. Hyaluronic Acid-Curcumin Conjugate. Let $G_{n}=$ HAC denote the molecular graph of hyaluronic acid-curcumin conjugates with the linear iteration $n$ units. The corresponding molecular graphs of hyaluronic acid-curcumin conjugates for $n=1$ and 3 are shown in Figure 3. 
TABLE 1: Various ve-degree topological indices derived from the general ve-inverse sum indeg index.

\begin{tabular}{|c|c|}
\hline Topological index & Corresponding $\operatorname{ISI}_{(\alpha, \beta)}^{\mathrm{ve}}(G)$ \\
\hline First ve-degree Zagreb beta index, $M_{1}^{\beta \mathrm{ve}}(G)=\sum_{u v \in E(G)}\left(d_{\mathrm{ve}}(u)+d_{\mathrm{ve}}(v)\right)$ & $\operatorname{ISI}_{(0,1)}^{v e}(G)$ \\
\hline Second ve-degree Zagreb index, $M_{2}^{\mathrm{ve}}(G)=\sum_{u v \in E(G)} d_{\mathrm{ve}}(u) d_{\mathrm{ve}}(v)$ & $\operatorname{ISI}_{(1,0)}^{v e(1)}(G)$ \\
\hline Ve-degree Randić index, $R^{\mathrm{ve}}(G)=\sum_{u v \in E(G)}\left(d_{\mathrm{ve}}(u) d_{\mathrm{ve}}(v)\right)^{-1 / 2}$ & $\operatorname{ISI}_{(-(1 / 2), 0)}^{v e}(G)$ \\
\hline Ve-degree sum-connectivity index, $\chi^{\mathrm{ve}}(G)=\sum_{u v \in E(G)}\left(d_{\mathrm{ve}}(u)+d_{\mathrm{ve}}(v)\right)^{-1 / 2}$ & $\operatorname{ISI}_{(0,-(1 / 2))}^{v e}(G)$ \\
\hline Ve-degree harmonic index, $H^{\mathrm{ve}}(G)=\sum_{u v \in E(G)}\left(2 /\left(d_{\mathrm{ve}}(u)+d_{\mathrm{ve}}(v)\right)\right)$ & $2 \operatorname{ISI}_{(0,-1)}^{v e}(G)$ \\
\hline First hyper ve-degree index, $\operatorname{HM}_{1}^{\mathrm{ve}}(G)=\sum_{u v \in E(G)}\left(d_{\mathrm{ve}}(u)+d_{\mathrm{ve}}(v)\right)^{2}$ & $\operatorname{ISI}_{(0,2)}^{v e}((G)$ \\
\hline Second hyper ve-degree index, $\operatorname{HM}_{2}^{\mathrm{ve}}(G)=\sum_{u v \in E(G)}\left(d_{\mathrm{ve}}(u) d_{\mathrm{ve}}(v)\right)^{2}$ & $\operatorname{ISI}_{(2,0)}^{v e}(G)$ \\
\hline Redefined third ve-degree Zagreb index, $\operatorname{ReZG}_{3}^{\mathrm{ve}}(G)=\sum_{u v \in E(G)} d_{\mathrm{ve}}(u) d_{\mathrm{ve}}(v)\left(d_{\mathrm{ve}}(u)+d_{\mathrm{ve}}(v)\right)$ & $\operatorname{ISI}_{(1,1)}^{v e r}(G)$ \\
\hline Ve-degree geometric-arithmetic index, $\mathrm{GA}^{\mathrm{ve}}(G)=\sum_{u v \in E(G)}\left(2 \sqrt{d_{\mathrm{ve}}(u) d_{\mathrm{ve}}(v)} /\left(d_{\mathrm{ve}}(u)+d_{\mathrm{ve}}(v)\right)\right)$ & $2 \operatorname{ISI}_{((1 / 2),-1)}^{v e(G)}$ \\
\hline Ve-degree arithmetic-geometric index, $\mathrm{AG}^{\mathrm{ve}}(G)=\sum_{u v \in E(G)}\left(\left(d_{\mathrm{ve}}(u)+d_{\mathrm{ve}}(v)\right) / 2 \sqrt{d_{\mathrm{ve}}(u) d_{\mathrm{ve}}(v)}\right)$ & $(1 / 2) \operatorname{ISI}_{(-(1 / 2), 1)}^{v e}(G)$ \\
\hline Ve-degree inverse sum indeg index, $\operatorname{ISI}^{\mathrm{ve}}(G)=\sum_{u v \in E(G)}\left(\left(d_{\mathrm{ve}}(u) d_{\mathrm{ve}}(v)\right) /\left(d_{\mathrm{ve}}(u)+d_{\mathrm{ve}}(v)\right)\right)$ & $\operatorname{ISI}_{(1,-1)}^{v e}(G)$ \\
\hline
\end{tabular}

TABLE 2: Various $v e$-degree topological indices derived from the general ve-Zagreb index.

\begin{tabular}{lc}
\hline Topological index & Corresponding $M_{\alpha}^{v e}(G)$ \\
\hline Total ve-degree $T^{v e}(G)=\sum_{u \in V(G)} d_{v e}(u)$ & $M_{1}^{v e}(G)$ \\
First ve-degree Zagreb alpha index, $M_{1}^{\alpha v e}(G)=\sum_{u \in V(G)} d_{\mathrm{ve}}(u)^{2}$ & $M_{2}^{v e}(G)$ \\
$F_{1}$-ve-degree index, $F_{1}^{\mathrm{ve}}(G)=\sum_{u \in V(G)} d_{\mathrm{ve}}(u)^{3}$ & $M_{3}^{v e}(G)$ \\
Inverse ve-degree index, $I D^{v e}(G)=\sum_{u \in V(G)} 1 /\left(d_{v e}(u)\right)$ & $M_{-1}^{v e}(G)$ \\
Zeroth order ve-degree index, $Z D^{v e}(G)=\sum_{u \in V(G)} 1 / \sqrt{\left(d_{v e}(u)\right)}$ & $M_{-(1 / 2)}^{v e}(G)$ \\
Modified first ve-degree index, $m M_{1}^{v e}(G)=\sum_{u \in V(G)} 1 /\left(d_{v e}(u)^{2}\right)$ & $M_{-2}^{v e}(G)$
\end{tabular}

TABLE 3: Various ev-degree topological indices derived from the general $e v$-degree index.

\begin{tabular}{lc}
\hline Topological index & $\begin{array}{c}\text { Corresponding } \\
M_{\alpha}^{\mathrm{ev}}(G)\end{array}$ \\
\hline $\begin{array}{l}\text { Total } e v \text {-degree } T^{e v}(G)=\sum_{e \in E(G)} d_{e v}(e) \\
e v \text {-degree Zagreb index, }\end{array}$ & $M_{1}^{e v}(G)$ \\
$M^{\text {ev }}(G)=\sum_{e \in E(G)} d_{e v}(e)^{2}$ & $M_{2}^{e v}(G)$ \\
$F$-ev-degree index, $F^{e v}(G)=\sum_{e \in E(G)} d_{e v}(e)^{3}$ & $M_{3}^{e v}(G)$ \\
Modified $e v$-degree Zagreb index, & $M_{-2}^{e v}(G)$ \\
$m M^{e v}(G)=\sum_{e \in E(G)} 1 /\left(d_{e v}(e)^{2}\right)$ & $M_{-1}^{e v}(G)$ \\
$e v$-degree inverse index, & $M_{-(1 / 2)}^{e v}(G)$ \\
$I D^{e v}(G)=\sum_{e \in E(G)} 1 /\left(d_{e v}(e)\right)$ & $M_{(1 / 2)}^{e v}(G)$ \\
$e v$-degree Randić index, & \\
$R^{e v}(G)=\sum_{e \in E(G)} d_{e v}(e)^{-}(1 / 2)$ & \\
Reciprocal $e v$-degree Randic index, & \\
$R R^{e v}(G)=\sum_{e \in E(G)} d_{e v}(e)^{1 / 2}$ & \\
\hline
\end{tabular}

Let us start our discussion with the partitioning of edge set $E$ (HAC) on the basic of ve-degree of vertices. By molecular graph structure analysis and observation, we note that the edge set of HAC can be divided into seventeen edge groups based on ve-degree of its end vertices as summarized in Table 4.

Now, we proceed to establish the expression for the general ve-inverse sum indeg index in the following theorem.

Theorem 1. The general ve-inverse sum indeg index $\operatorname{ISI}_{(\alpha, \beta)}^{v e}$ of HA-curcumin conjugate is given by

From the molecular structure of HAC, it is easy to conclude that $|V(\mathrm{HAC})|=52 n+1$ and $|E(\mathrm{HAC})|=56 n$.

$$
\begin{aligned}
\operatorname{ISI}_{(\alpha, \beta)}^{\mathrm{ve}}(\mathrm{HAC})= & (3 n+1)[8]^{\alpha}[6]^{\beta}+2 n[12]^{\alpha}[7]^{\beta}+2 n[15]^{\alpha}[8]^{\beta}+2 n[18]^{\alpha}[9]^{\beta} \\
& +3 n[21]^{\alpha}[10]^{\beta}+(6 n+1)[25]^{\alpha}[10]^{\beta}+1 .[32]^{\alpha}[12]^{\beta}+(9 n-2)[42]^{\alpha}[13]^{\beta}+(5 n-2)[48]^{\alpha}[14]^{\beta} \\
& +3 n[28]^{\alpha}[11]^{\beta}+(n+2)[35]^{\alpha}[12]^{\beta}+n[24]^{\alpha}[10]^{\beta}+3 n[36]^{\alpha}[12]^{\beta}+7 n[30]^{\alpha}[11]^{\beta}+(2 n-1)[64]^{\alpha}[16]^{\beta} \\
& +3 n[56]^{\alpha}[15]^{\beta}+4 n[49]^{\alpha}[14]^{\beta} .
\end{aligned}
$$

Proof. Clearly, from Table 4, we have 


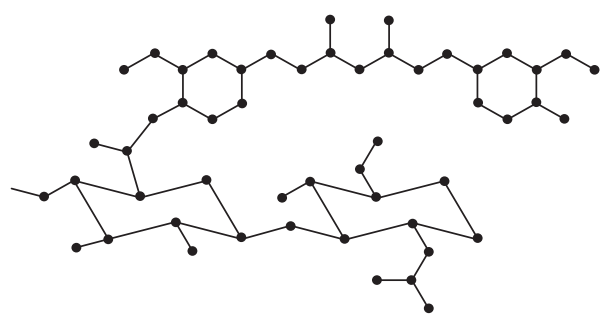

(a)

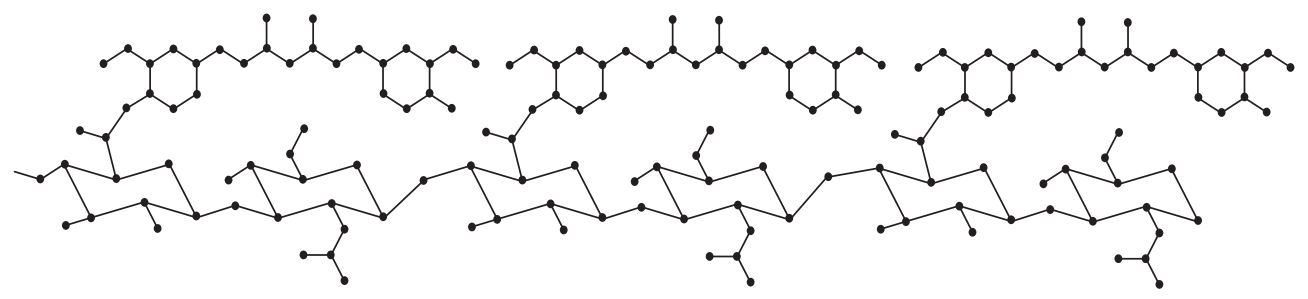

(b)

FIGURE 3: Corresponding molecular graph of hyaluronic acid-curcumin conjugates HAC; $n=1, n=3$.

TABLE 4: The ve-degree of the end vertices of edges of HAC.

\begin{tabular}{lcc}
\hline Ve-degree of its end vertices $\left(d_{\mathrm{ve}}(u), d_{\mathrm{ve}}(v)\right)$ & Number of edges (frequency) & $E_{2,4}$ \\
\hline$(2,4)$ & $3 n+1$ & $E_{2,4}$ \\
$(3,4)$ & $2 n$ & $E_{3,4}$ \\
$(3,5)$ & $2 n$ & $E_{3,5}$ \\
$(3,6)$ & $2 n$ & $E_{3,6}$ \\
$(3,7)$ & $3 n$ & $E_{3,7}$ \\
$(5,5)$ & $6 n+1$ & $E_{5,5}$ \\
$(4,8)$ & 1 & $E_{4,8}$ \\
$(6,7)$ & $9 n-2$ & $E_{6,7}$ \\
$(6,8)$ & $5 n-2$ & $E_{6,8}$ \\
$(4,7)$ & $3 n$ & $E_{4,7}$ \\
$(5,7)$ & $n+2$ & $E_{5,7}$ \\
$(4,6)$ & $N$ & $E_{4,6}$ \\
$(6,6)$ & $3 n$ & $E_{6,6}$ \\
$(5,6)$ & $7 n$ & $E_{5,6}$ \\
$(8,8)$ & $2 n-1$ & $E_{8,8}$ \\
$(7,8)$ & $3 n$ & $E_{7,8}$ \\
$(7,7)$ & $4 n$ & $E_{7,7}$ \\
\hline
\end{tabular}

$$
\begin{aligned}
& \left|E_{2,4}(\mathrm{HAC})\right|=3 n+1, \\
& \mid E_{3,4} \text { (HAC) } \mid=2 n, \\
& \left|E_{3,5}(\mathrm{HAC})\right|=2 n, \\
& \left|E_{3,6}(\mathrm{HAC})\right|=2 n, \\
& \left|E_{3,7}(\mathrm{HAC})\right|=3 n, \\
& \mid E_{5,5} \text { (HAC) } \mid=6 n+1, \\
& \mid E_{4,8} \text { (HAC) } \mid=1, \\
& \left|E_{6,7}(\mathrm{HAC})\right|=9 n-2,
\end{aligned}
$$

$$
\begin{aligned}
& \left|E_{6,8}(\mathrm{HAC})\right|=5 n-2, \\
& \left|E_{4,7}(\mathrm{HAC})\right|=3 n, \\
& \left|E_{5,7}(\mathrm{HAC})\right|=n+2, \\
& \left|E_{4,6}(\mathrm{HAC})\right|=n, \\
& \left|E_{6,6}(\mathrm{HAC})\right|=3 n, \\
& \left|E_{5,6}(\mathrm{HAC})\right|=7 n, \\
& \left|E_{8,8}(\mathrm{HAC})\right|=2 n-1, \\
& \left|E_{7,8}(\mathrm{HAC})\right|=3 n, \\
& \left|E_{7,7}(\mathrm{HAC})\right|=4 n .
\end{aligned}
$$


Now, applying the definition of the general ve-ISI index, $\operatorname{ISI}_{(\alpha, \beta)}^{\mathrm{ve}}$, we have

$$
\begin{aligned}
& \operatorname{ISI}_{(\alpha, \beta)}^{\mathrm{ve}}(G)=\sum_{u v \in E(G)}\left[d_{\mathrm{ve}}(u) d_{\mathrm{ve}}(v)\right]^{\alpha}\left[d_{\mathrm{ve}}(u)+d_{\mathrm{ve}}(v)\right]^{\beta} \\
& \operatorname{ISI}_{(\alpha, \beta)}(\mathrm{HAC})=\sum_{u v \in E_{2,4}(\mathrm{HAC})}\left[d_{\mathrm{ve}}(u) d_{\mathrm{ve}}(v)\right]^{\alpha}\left[d_{\mathrm{ve}}(u)+d_{\mathrm{ve}}(v)\right]^{\beta}+\sum_{u v \in E_{3,4}(\mathrm{HAC})}\left[d_{\mathrm{ve}}(u) d_{\mathrm{ve}}(v)\right]^{\alpha}\left[d_{\mathrm{ve}}(u)+d_{\mathrm{ve}}(v)\right]^{\beta} \\
& +\sum_{u v \in E_{3,5}(\mathrm{HAC})}\left[d_{\mathrm{ve}}(u) d_{\mathrm{ve}}(v)\right]^{\alpha}\left[d_{\mathrm{ve}}(u)+d_{\mathrm{ve}}(v)\right]^{\beta}+\sum_{u v \in E_{3,6}(\mathrm{HAC})}\left[d_{\mathrm{ve}}(u) d_{\mathrm{ve}}(v)\right]^{\alpha}\left[d_{\mathrm{ve}}(u)+d_{\mathrm{ve}}(v)\right]^{\beta} \\
& +\sum_{u v \in E_{3,7}(\mathrm{HAC})}\left[d_{\mathrm{ve}}(u) d_{\mathrm{ve}}(v)\right]^{\alpha}\left[d_{\mathrm{ve}}(u)+d_{\mathrm{ve}}(v)\right]^{\beta}+\sum_{u v \in E_{5,5}(\mathrm{HAC})}\left[d_{\mathrm{ve}}(u) d_{\mathrm{ve}}(v)\right]^{\alpha}\left[d_{\mathrm{ve}}(u)+d_{\mathrm{ve}}(v)\right]^{\beta} \\
& +\sum_{u v \in E_{4,8}(\mathrm{HAC})}\left[d_{\mathrm{ve}}(u) d_{\mathrm{ve}}(v)\right]^{\alpha}\left[d_{\mathrm{ve}}(u)+d_{\mathrm{ve}}(v)\right]^{\beta}+\sum_{u v \in E_{6,7}(\mathrm{HAC})}\left[d_{\mathrm{ve}}(u) d_{\mathrm{ve}}(v)\right]^{\alpha}\left[d_{\mathrm{ve}}(u)+d_{\mathrm{ve}}(v)\right]^{\beta} \\
& +\sum_{u v \in E_{6,8}(\mathrm{HAC})}\left[d_{\mathrm{ve}}(u) d_{\mathrm{ve}}(v)\right]^{\alpha}\left[d_{\mathrm{ve}}(u)+d_{\mathrm{ve}}(v)\right]^{\beta}+\sum_{u v \in E_{4,7}(\mathrm{HAC})}\left[d_{\mathrm{ve}}(u) d_{\mathrm{ve}}(v)\right]^{\alpha}\left[d_{\mathrm{ve}}(u)+d_{\mathrm{ve}}(v)\right]^{\beta} \\
& +\sum_{u v \in E_{5,7}(\mathrm{HAC})}\left[d_{\mathrm{ve}}(u) d_{\mathrm{ve}}(v)\right]^{\alpha}\left[d_{\mathrm{ve}}(u)+d_{\mathrm{ve}}(v)\right]^{\beta}+\sum_{u v \in E_{4,6}(\mathrm{HAC})}\left[d_{\mathrm{ve}}(u) d_{\mathrm{ve}}(v)\right]^{\alpha}\left[d_{\mathrm{ve}}(u)+d_{\mathrm{ve}}(v)\right]^{\beta} \\
& +\sum_{u v \in E_{6,6}(\mathrm{HAC})}\left[d_{\mathrm{ve}}(u) d_{\mathrm{ve}}(v)\right]^{\alpha}\left[d_{\mathrm{ve}}(u)+d_{\mathrm{ve}}(v)\right]^{\beta}+\sum_{u v \in E_{5,6}(\mathrm{HAC})}\left[d_{\mathrm{ve}}(u) d_{\mathrm{ve}}(v)\right]^{\alpha}\left[d_{\mathrm{ve}}(u)+d_{\mathrm{ve}}(v)\right]^{\beta} \\
& +\sum_{u v \in E_{8,8}(\mathrm{HAC})}\left[d_{\mathrm{ve}}(u) d_{\mathrm{ve}}(v)\right]^{\alpha}\left[d_{\mathrm{ve}}(u)+d_{\mathrm{ve}}(v)\right]^{\beta}+\sum_{u v \in E_{7,8}(\mathrm{HAC})}\left[d_{\mathrm{ve}}(u) d_{\mathrm{ve}}(v)\right]^{\alpha}\left[d_{\mathrm{ve}}(u)+d_{\mathrm{ve}}(v)\right]^{\beta} \\
& +\sum_{u v \in E_{7,7}(\mathrm{HAC})}\left[d_{\mathrm{ve}}(u) d_{\mathrm{ve}}(v)\right]^{\alpha}\left[d_{\mathrm{ve}}(u)+d_{\mathrm{ve}}(v)\right]^{\beta} \\
& =\left|E_{2,4}(\mathrm{HAC})\right|[(2)(4)]^{\alpha}[2+4]^{\beta}+\left|E_{3,4}(\mathrm{HAC})\right|[(3)(4)]^{\alpha}[3+4]^{\beta} \\
& +\left|E_{3,5}(\mathrm{HAC})\right|[(3)(5)]^{\alpha}[3+5]^{\beta}+\left|E_{3,6}(\mathrm{HAC})\right|[(3)(6)]^{\alpha}[3+6]^{\beta} \\
& +\left|E_{3,7}(\mathrm{HAC})\right|[(3)(7)]^{\alpha}[3+7]^{\beta}+\left|E_{5,5}(\mathrm{HAC})\right|[(5)(5)]^{\alpha}[5+5]^{\beta}+\left|E_{4,8}(\mathrm{HAC})\right|[(4)(8)]^{\alpha}[4+8]^{\beta} \\
& +\left|E_{6,7}(\mathrm{HAC})\right|[(6)(7)]^{\alpha}[6+7]^{\beta}+\left|E_{6,8}(\mathrm{HAC})\right|[(6)(8)]^{\alpha}[6+8]^{\beta}+\left|E_{4,7}(\mathrm{HAC})\right|[(4)(7)]^{\alpha}[4+7]^{\beta} \\
& +\left|E_{5,7}(\mathrm{HAC})\right|[(5)(7)]^{\alpha}[5+7]^{\beta}+\left|E_{4,6}(\mathrm{HAC})\right|[(4)(6)]^{\alpha}[4+6]^{\beta}+\left|E_{6,6}(\mathrm{HAC})\right|[(6)(6)]^{\alpha}[6+6]^{\beta} \\
& +\left|E_{5,6}(\mathrm{HAC})\right|[(5)(6)]^{\alpha}[5+6]^{\beta}+\left|E_{8,8}(\mathrm{HAC})\right|[(8)(8)]^{\alpha}[8+8]^{\beta}+\left|E_{7,8}(\mathrm{HAC})\right|[(7)(8)]^{\alpha}[7+8]^{\beta} \\
& +\left|E_{7,7}(\mathrm{HAC})\right|[(7)(7)]^{\alpha}[7+7]^{\beta} \\
& =(3 n+1)[8]^{\alpha}[6]^{\beta}+2 n[12]^{\alpha}[7]^{\beta}+2 n[15]^{\alpha}[8]^{\beta}+2 n[18]^{\alpha}[9]^{\beta} \\
& +3 n[21]^{\alpha}[10]^{\beta}+(6 n+1)[25]^{\alpha}[10]^{\beta}+1 \cdot[32]^{\alpha}[12]^{\beta}+(9 n-2)[42]^{\alpha}[13]^{\beta} \\
& +(5 n-2)[48]^{\alpha}[14]^{\beta}+3 n[28]^{\alpha}[11]^{\beta}+(n+2)[35]^{\alpha}[12]^{\beta}+n[24]^{\alpha}[10]^{\beta} \\
& +3 n[36]^{\alpha}[12]^{\beta}+7 n[30]^{\alpha}[11]^{\beta}+(2 n-1)[64]^{\alpha}[16]^{\beta}+3 n[56]^{\alpha}[15]^{\beta}+4 n[49]^{\alpha}[14]^{\beta} \text {. }
\end{aligned}
$$


Hence, the theorem.

(i) $M_{1}^{\beta v e}(H A C)=644 n-18$

Next, we retrieve exact values of the most well-known vedegree-based indices of HA-curcumin conjugate.

(ii) $M_{2}^{v e}(H A C)=1898 n-109$

(iii)

Corollary 1. Let HAC be the molecular graph of HA-curcumin conjugate; then,

$$
\begin{aligned}
R^{v e}(\mathrm{HAC})= & \left(\frac{13 \sqrt{2}}{12}+\frac{3 \sqrt{3}}{4}+\frac{2}{\sqrt{15}}+\frac{3}{\sqrt{21}}+\frac{9}{\sqrt{42}}+\frac{3}{2 \sqrt{7}}+\frac{1}{\sqrt{35}}+\frac{1}{2 \sqrt{6}}+\frac{7}{\sqrt{30}}+\frac{3}{2 \sqrt{14}}+\frac{353}{140}\right) n \\
& +\left(\frac{3}{2 \sqrt{2}}+\frac{1}{5}-\frac{2}{\sqrt{42}}-\frac{2}{4 \sqrt{3}}+\frac{2}{\sqrt{35}}-\frac{1}{8}\right) .
\end{aligned}
$$

(iv)

$$
\begin{aligned}
\chi^{\mathrm{ve}}(\mathrm{HAC})= & \left(\frac{3}{\sqrt{6}}+\frac{2}{\sqrt{7}}+\frac{2}{\sqrt{8}}+\frac{2}{\sqrt{9}}+\frac{10}{\sqrt{10}}+\frac{10}{\sqrt{11}}+\frac{4}{\sqrt{12}}+\frac{9}{\sqrt{13}}+\frac{9}{\sqrt{14}}+\frac{3}{\sqrt{15}}+\frac{2}{\sqrt{16}}\right) n \\
& +\left(\frac{1}{\sqrt{6}}+\frac{1}{\sqrt{10}}+\frac{3}{\sqrt{12}}-\frac{2}{\sqrt{13}}-\frac{2}{\sqrt{14}}-\frac{1}{\sqrt{16}}\right) .
\end{aligned}
$$

(v) $H^{v e}(H A C)=(1859647 / 180180) n-(3439 / 10920)$

(vi) $H M_{1}^{v e}(H A C)=7754 n-418$

(vii) $H M_{2}^{v e}(H A C)=74892 n-8069$ (viii) $\operatorname{ReZG}_{3}^{v e}(H A C)=23782 n-1938$

(ix)

$$
\begin{aligned}
\mathrm{GA}^{\mathrm{ve}}(\mathrm{HAC})= & \left.\frac{10 \sqrt{2}}{3}+\frac{28 \sqrt{3}}{7}+\frac{18 \sqrt{42}}{13}+\frac{\sqrt{15}}{2}+\frac{3 \sqrt{21}}{5}+\frac{12 \sqrt{7}}{11}+\frac{\sqrt{35}}{6}+\frac{2 \sqrt{6}}{5}+\frac{4 \sqrt{30}}{11}+\frac{4 \sqrt{14}}{5}+15\right) n \\
& +\frac{4 \sqrt{2}}{3}-\frac{4 \sqrt{42}}{13}-\frac{8 \sqrt{3}}{7}+\frac{\sqrt{35}}{3}
\end{aligned}
$$

(x)

$\mathrm{AG}^{\mathrm{ve}}(\mathrm{HAC})=\left(\frac{15}{2 \sqrt{2}}+\frac{49}{4 \sqrt{3}}+\frac{5}{2 \sqrt{6}}+\frac{33}{4 \sqrt{7}}+\frac{45}{4 \sqrt{14}}+\frac{8}{\sqrt{15}}+\frac{15}{\sqrt{21}}+\frac{77}{\sqrt{30}}+\frac{6}{\sqrt{35}}+\frac{117}{2 \sqrt{42}}\right) n+\frac{3}{\sqrt{2}}-\frac{7}{2 \sqrt{3}}+\frac{12}{\sqrt{35}}-\frac{13}{\sqrt{42}}$

(xi) $\operatorname{ISI}^{v e}(H A C)=(4712977 / 30030) n-(1361 / 273)$

Proof. From Theorem 1, we have

$$
\begin{aligned}
\operatorname{ISI}_{(\alpha, \beta)}^{\mathrm{ve}}(\mathrm{HAC})= & (3 n+1)[8]^{\alpha}[6]^{\beta}+2 n[12]^{\alpha}[7]^{\beta}+2 n[15]^{\alpha}[8]^{\beta}+2 n[18]^{\alpha}[9]^{\beta} \\
& +3 n[21]^{\alpha}[10]^{\beta}+(6 n+1)[25]^{\alpha}[10]^{\beta}+1 .[32]^{\alpha}[12]^{\beta}+(9 n-2)[42]^{\alpha}[13]^{\beta} \\
& +(5 n-2)[48]^{\alpha}[14]^{\beta}+3 n[28]^{\alpha}[11]^{\beta}+(n+2)[35]^{\alpha}[12]^{\beta}+n[24]^{\alpha}[10]^{\beta} \\
& +3 n[36]^{\alpha}[12]^{\beta}+7 n[30]^{\alpha}[11]^{\beta}+(2 n-1)[64]^{\alpha}[16]^{\beta}+3 n[56]^{\alpha}[15]^{\beta}+4 n[49]^{\alpha}[14]^{\beta}
\end{aligned}
$$


(i) Put $\alpha=0$ and $\beta=1$ in equation (25); then, $\operatorname{ISI}_{(0,1)}^{\mathrm{ve}}(\mathrm{HAC})=M_{1}^{\beta \mathrm{ve}}(\mathrm{HAC})=644 n-18$ is the first ve-degree Zagreb beta index. (ii) If $\alpha=1$ and $\beta=0$, then $\operatorname{ISI}_{(1,0)}^{\mathrm{ve}}(\mathrm{HAC})=$ $M_{2}^{\mathrm{ve}}$ (HAC) is the second ve-degree Zagreb index and $M_{2}^{\mathrm{ve}}(G)=1898 n-109$

(iii) For $\alpha=-(1 / 2)$ and $\beta=0$, equation (25) gives vedegree Randić index, i.e.,

$$
\begin{aligned}
& \operatorname{ISI}_{(-(1 / 2), 0)}^{\mathrm{ve}}(\mathrm{HAC})= \\
& R^{\mathrm{ve}}(\mathrm{HAC})=\left(\frac{13 \sqrt{2}}{12}+\frac{3 \sqrt{3}}{4}+\frac{2}{\sqrt{15}}+\frac{3}{\sqrt{21}}+\frac{9}{\sqrt{42}}+\frac{3}{2 \sqrt{7}}+\frac{1}{\sqrt{35}}+\frac{1}{2 \sqrt{6}}+\frac{7}{\sqrt{30}}+\frac{3}{2 \sqrt{14}}+\frac{353}{140}\right) n \\
& +\left(\frac{3}{2 \sqrt{2}}+\frac{1}{5}-\frac{2}{\sqrt{42}}-\frac{2}{4 \sqrt{3}}+\frac{2}{\sqrt{35}}-\frac{1}{8}\right) .
\end{aligned}
$$

(iv) If $\alpha=0$ and $\beta=-(1 / 2)$, then $\operatorname{ISI}_{(0,-(1 / 2))}^{\mathrm{ve}}$ $(\mathrm{HAC})=\chi^{\mathrm{ve}}(\mathrm{HAC})$, i.e., ve-degree sum-connectivity index

$$
\begin{aligned}
\chi^{\text {ve }}(\mathrm{HAC})= & \left(\frac{3}{\sqrt{6}}+\frac{2}{\sqrt{7}}+\frac{2}{\sqrt{8}}+\frac{2}{\sqrt{9}}+\frac{10}{\sqrt{10}}+\frac{10}{\sqrt{11}}+\frac{4}{\sqrt{12}}+\frac{9}{\sqrt{13}}+\frac{9}{\sqrt{14}}+\frac{3}{\sqrt{15}}+\frac{2}{\sqrt{16}}\right) n \\
& +\left(\frac{1}{\sqrt{6}}+\frac{1}{\sqrt{10}}+\frac{3}{\sqrt{12}}-\frac{2}{\sqrt{13}}-\frac{2}{\sqrt{14}}-\frac{1}{\sqrt{16}}\right) .
\end{aligned}
$$

(v) Put $\alpha=0$ and $\beta=-1$ in equation (25) and then multiply this equation by 2 ; we get the ve-degree harmonic index

$$
\operatorname{ISI}_{(0,-1)}^{\mathrm{ve}}(\text { HAC })=H^{\mathrm{ve}}(\text { HAC })=\frac{1859647}{180180} n-\frac{3439}{10920} \text {. }
$$

(vi) If $\alpha=0$ and $\beta=2$, then we have the first hyper vedegree Zagreb index

$$
\operatorname{ISI}_{(0,2)}^{\mathrm{ve}}(\mathrm{HAC})=\mathrm{HM}_{1}^{\mathrm{ve}}(\mathrm{HAC})=7754 n-418 .
$$

(vii) Substituting $\alpha=2$ and $\beta=0$, we get the second hyper ve-degree Zagreb index

$$
\operatorname{ISI}_{(2,0)}^{\mathrm{ve}}(\mathrm{HAC})=\mathrm{HM}_{2}^{\mathrm{ve}}(\mathrm{HAC})=74892 n-8069
$$

(viii) If $\alpha=1$ and $\beta=1$, then we have redefined the third ve-degree Zagreb index as

$$
\operatorname{ISI}_{(1,1)}^{\mathrm{ve}}(\mathrm{HAC})=\operatorname{ReZG}_{3}^{\mathrm{ve}}(\mathrm{HAC})=23782 n-1938 .
$$

(ix) If we take $\alpha=1 / 2$ and $\beta=-1$ in equation (25) and then multiply result by 2 , this gives the ve-degree geometric-arithmetic index $\mathrm{GA}^{\mathrm{ve}}$ (HAC) as

$$
\begin{aligned}
\mathrm{GA}^{\mathrm{ve}}(\mathrm{HAC})= & \left.\frac{10 \sqrt{2}}{3}+\frac{28 \sqrt{3}}{7}+\frac{18 \sqrt{42}}{13}+\frac{\sqrt{15}}{2}+\frac{3 \sqrt{21}}{5}+\frac{12 \sqrt{7}}{11}+\frac{\sqrt{35}}{6}+\frac{2 \sqrt{6}}{5}+\frac{4 \sqrt{30}}{11}+\frac{4 \sqrt{14}}{5}+15\right) n \\
& +\frac{4 \sqrt{2}}{3}-\frac{4 \sqrt{42}}{13}-\frac{8 \sqrt{3}}{7}+\frac{\sqrt{35}}{3} .
\end{aligned}
$$


(x) By taking $\alpha=-(1 / 2)$ and $\beta=1$ in equation (25) and multiplying by $1 / 2$, we get

$\mathrm{AG}^{\mathrm{ve}}(\mathrm{HAC})=\left(\frac{15}{2 \sqrt{2}}+\frac{49}{4 \sqrt{3}}+\frac{5}{2 \sqrt{6}}+\frac{33}{4 \sqrt{7}}+\frac{45}{4 \sqrt{14}}+\frac{8}{\sqrt{15}}+\frac{15}{\sqrt{21}}+\frac{77}{\sqrt{30}}+\frac{6}{\sqrt{35}}+\frac{117}{2 \sqrt{42}}\right) n+\frac{3}{\sqrt{2}}-\frac{7}{2 \sqrt{3}}+\frac{12}{\sqrt{35}}-\frac{13}{\sqrt{42}}$

(xi) If $\alpha=1$ and $\beta=-1$, then we have the ve-degree inverse sum indeg index

$$
\mathrm{ISI}^{\mathrm{ve}}(\mathrm{HAC})=\frac{4712977}{30030} n-\frac{1361}{273} \text {. }
$$

Now, we partition the vertex set of HAC, i.e., V(HAC) into seven vertex groups on the basis of ve-degree of vertex as given in Table 5 .

Theorem 2. The general ve-Zagreb index $M_{\alpha}^{v e}$ of HA-curcumin conjugate is given by

$$
\begin{aligned}
M_{\alpha}^{\mathrm{ve}}(\mathrm{HAC})= & (3 n+1)[2]^{\alpha}+9 n[3]^{\alpha}+(4 n+1)[4]^{\alpha} \\
& +(10 n+2)[5]^{\alpha}+(13 n-2)[6]^{\alpha} \\
& +9 n[7]^{\alpha}+(4 n-1)[8]^{\alpha} .
\end{aligned}
$$

Proof. From Table 5, we have

$$
\begin{aligned}
& \left|V_{2}(\mathrm{HAC})\right|=3 n+1, \\
& \left|V_{3}(\mathrm{HAC})\right|=9 n, \\
& \left|V_{4}(\mathrm{HAC})\right|=4 n+1, \\
& \left|V_{5}(\mathrm{HAC})\right|=10 n+2, \\
& \left|V_{6}(\mathrm{HAC})\right|=13 n-2, \\
& \left|V_{7}(\mathrm{HAC})\right|=9 n, \\
& \left|V_{8}(\mathrm{HAC})\right|=4 n-1 .
\end{aligned}
$$
have

$$
\begin{aligned}
M_{\alpha}^{\mathrm{ve}}(G)= & \sum_{u \in V(G)}\left[d_{\mathrm{ve}}(u)\right]^{\alpha}, \\
M_{\alpha}^{\mathrm{ve}}(\mathrm{HAC})= & \sum_{u \in V_{2}(\mathrm{HAC})}\left[d_{\mathrm{ve}}(u)\right]^{\alpha}+\sum_{u \in V_{3}(\mathrm{HAC})}\left[d_{\mathrm{ve}}(u)\right]^{\alpha}+\sum_{u \in V_{4}(\mathrm{HAC})}\left[d_{\mathrm{ve}}(u)\right]^{\alpha} \\
& +\sum_{u \in V_{5}(\mathrm{HAC})}\left[d_{\mathrm{ve}}(u)\right]^{\alpha}+\sum_{u \in V_{6}(\mathrm{HAC})}\left[d_{\mathrm{ve}}(u)\right]^{\alpha}+\sum_{u \in V_{7}(\mathrm{HAC})}\left[d_{\mathrm{ve}}(u)\right]^{\alpha} \\
& +\sum_{u \in V_{8}(\mathrm{HAC})}\left[d_{\mathrm{ve}}(u)\right]^{\alpha}, \\
M_{\alpha}^{\mathrm{ve}}(\mathrm{HAC})= & \left|V_{2}(\mathrm{HAC})\right|[2]^{\alpha}+\left|V_{3}(\mathrm{HAC})\right|[3]^{\alpha}+\left|V_{4}(\mathrm{HAC})\right|[4]^{\alpha}+\left|V_{5}(\mathrm{HAC})\right|[5]^{\alpha}+\left|V_{6}(\mathrm{HAC})\right|[6]^{\alpha}+\left|V_{7}(\mathrm{HAC})\right|[7]^{\alpha} \\
& +\left|V_{8}(\mathrm{HAC})\right|[8]^{\alpha}, \\
M_{\alpha}^{\mathrm{ve}}(\mathrm{HAC})= & (3 n+1)[2]^{\alpha}+9 n[3]^{\alpha}+(4 n+1)[4]^{\alpha}+(10 n+2)[5]^{\alpha}+(13 n-2)[6]^{\alpha}+9 n[7]^{\alpha}+(4 n-1)[8]^{\alpha} .
\end{aligned}
$$

Using Theorem 2, the following corollary can be obtained with little efforts.

Corollary 2. Let HAC be the molecular graph of HA-curcumin conjugate; then,
(i) $T^{v e}(H A C)=272 n-4$
(ii) $M_{1}^{\alpha v e}(H A C)=1572 n-66$

(iii) $F_{1}^{v e}(H A C)=9716 n-622$

(iv) $I^{v e}(H A C)=(481 / 42) n+(83 / 120)$

(v) $Z D^{v e}(H A C)=(2+(3 / \sqrt{2})+3 \sqrt{3}+(10 / \sqrt{5})+$ $(13 / \sqrt{6})+(9 / \sqrt{7})+\sqrt{2}) n+((1 / 2)+(1 / \sqrt{2})+$ $(2 / \sqrt{5})-(2 / \sqrt{6})-(1 / \sqrt{8}))$

(vi) $m M_{1}^{v e}(H A C)=(106097 / 35280) n+(4627 / 14400)$ 
TABLE 5: The ve-degree of the vertices of HAC.

\begin{tabular}{lcc}
\hline Ve-degree $d_{\mathrm{ve}}(u)$ & Number of vertices (frequency) & Vertex set $V_{i}$ \\
\hline 2 & $3 n+1$ & $V_{2}$ \\
3 & $9 n$ & $V_{3}$ \\
4 & $4 n+1$ & $V_{4}$ \\
5 & $10 n+2$ & $V_{5}$ \\
6 & $13 n-2$ & $V_{6}$ \\
7 & $9 n$ & $V_{7}$ \\
8 & $4 n-1$ & $V_{8}$ \\
\hline
\end{tabular}

Proof. From Theorem 2, we have

$$
\begin{aligned}
M_{\alpha}^{v e}(\mathrm{HAC})= & (3 n+1)[2]^{\alpha}+9 n[3]^{\alpha}+(4 n+1)[4]^{\alpha} \\
& +(10 n+2)[5]^{\alpha}+(13 n-2)[6]^{\alpha} \\
& +9 n[7]^{\alpha}+(4 n-1)[8]^{\alpha} .
\end{aligned}
$$

(i) Substituting $\alpha=1$ in equation (38) gives total vedegree $T^{\mathrm{ve}}(\mathrm{HAC})=272 n-4$

(ii) If $\alpha=2$ is substituted in equation (38), then the first ve-degree Zagreb alpha index is obtained as

$$
M_{2}^{\mathrm{ve}}(\mathrm{HAC})=M_{1}^{\alpha \mathrm{ve}}(\mathrm{HAC})=1572 n-66 .
$$

(iii) Substituting $\alpha=3$ in equation (38) gives the $F_{1}$-vedegree index, i.e.,

$$
M_{3}^{\mathrm{ve}}(\mathrm{HAC})=F_{1}^{\mathrm{ve}}(\mathrm{HAC})=9716 n-622 .
$$

(iv) For $\alpha=-1$, equation (38) gives the inverse ve-degree index, i.e.,

$$
M_{-1}^{\mathrm{ve}}(\mathrm{HAC})=\mathrm{ID}^{\mathrm{ve}}(\mathrm{HAC})=\frac{481}{42} n+\frac{83}{120} .
$$

(v) If $\alpha=-(1 / 2)$, then $M_{-(1 / 2)}^{\mathrm{ve}}$ (HAC)represents the zeroth order ve-degree index given by

$$
\begin{aligned}
\mathrm{ZD}^{\mathrm{ve}}(\mathrm{HAC})= & \left(2+\frac{3}{\sqrt{2}}+3 \sqrt{3}+\frac{10}{\sqrt{5}}+\frac{13}{\sqrt{6}}+\frac{9}{\sqrt{7}}+\sqrt{2}\right) n \\
& +\left(\frac{1}{2}+\frac{1}{\sqrt{2}}+\frac{2}{\sqrt{5}}-\frac{2}{\sqrt{6}}-\frac{1}{\sqrt{8}}\right) .
\end{aligned}
$$

(vi) If we put $\alpha=-2$ in equation (38), we get the modified first ve-degree index, i.e.,

$$
M_{-2}^{\mathrm{ve}}(\mathrm{HAC})=\mathrm{mM}_{1}^{\mathrm{ve}}(\mathrm{HAC})=\frac{106097}{35280} n+\frac{4627}{14400} .
$$

In Table 6, we partition the edge set of HAC into four edge groups on the basis of ev-degree of the edges.

Theorem 3. The general ev-degree index $M_{\alpha}^{e v}$ of $H A$ curcumin conjugate is given by

$$
\begin{aligned}
M_{\alpha}^{\mathrm{ev}}(\mathrm{HAC})= & (3 n+1)[3]^{\alpha}+(13 n+1)[4]^{\alpha}+(29 n-1)[5]^{\alpha} \\
& +(11 n-1)[6]^{\alpha} .
\end{aligned}
$$

Proof. Clearly,

$$
\begin{aligned}
& \left|E_{3}(\mathrm{HAC})\right|=3 n+1, \\
& \left|E_{4}(\mathrm{HAC})\right|=13 n+1, \\
& \left|E_{5}(\mathrm{HAC})\right|=29 n-1, \\
& \left|E_{6}(\mathrm{HAC})\right|=11 n-1 .
\end{aligned}
$$

Applying the definition of ev-degree index $M_{\alpha}^{\mathrm{ev}}$, we have

$$
\begin{aligned}
M_{\alpha}^{\mathrm{ev}}(G) & =\sum_{e \in E(G)}\left[d_{\mathrm{ev}}(e)\right]^{\alpha}, \\
M_{\alpha}^{e v}(\mathrm{HAC}) & =\sum_{e \in E_{3}(\mathrm{HAC})}\left[d_{\mathrm{ev}}(e)\right]^{\alpha}+\sum_{e \in E_{4}(\mathrm{HAC})}\left[d_{\mathrm{ev}}(e)\right]^{\alpha}+\sum_{e \in E_{5}(\mathrm{HAC})}\left[d_{\mathrm{ev}}(e)\right]^{\alpha}+\sum_{e \in E_{6}(\mathrm{HAC})}\left[d_{\mathrm{ev}}(e)\right]^{\alpha} \\
M_{\alpha}^{\mathrm{ev}}(\mathrm{HAC}) & =\left|E_{3}(\mathrm{HAC})\right|[3]^{\alpha}+\left|E_{4}(\mathrm{HAC})\right|[4]^{\alpha}+\left|E_{5}(\mathrm{HAC})\right|[5]^{\alpha}+\left|E_{6}(\mathrm{HAC})\right|[6]^{\alpha} \\
M_{\alpha}^{\mathrm{ev}}(\mathrm{HAC}) & =(3 n+1)[3]^{\alpha}+(13 n+1)[4]^{\alpha}+(29 n-1)[5]^{\alpha}+(11 n-1)[6]^{\alpha} .
\end{aligned}
$$

Next, corollary is immediate from Theorem 3.

Corollary 3. Let HAC be the molecular graph of HA-curcumin conjugate; then,

(i) $T^{e v}(H A C)=272 n-4$

(ii) $M^{\text {ev }}(H A C)=1356 n-36$

(iii) $F^{e v}(H A C)=6914 n-250$

(iv) $m M^{e v}($ HAC $)==(9401 / 3600) n+(127 / 1200)$

(v) $I D^{e v}(H A C)=(713 / 60) n+(13 / 60)$ (vi) $R^{e v}(H A C)=((13 / 2)+\sqrt{3}+(29 / \sqrt{5})+$

$(11 / \sqrt{6})) n+((1 / 2)+(1 / \sqrt{3})-(1 / \sqrt{5})-(1 / 6))$

(vii) $R R^{e v}(H A C)=(3 \sqrt{3}+29 \sqrt{5}+11 \sqrt{6}+26) n+$

$(\sqrt{3}-\sqrt{5}-\sqrt{6}+2)$

Proof. Substituting $\alpha=1,2,3,-2,-1,-(1 / 2),(1 / 2) \quad$ in equation (44), we get desired results.

2.2. Hyaluronic Acid-Paclitaxel Conjugates. Let $G_{n}=\mathrm{HAP}$ denote the molecular graph of hyaluronic acid-paclitaxel 
TABle 6: The ev-degree of the edges of HAC.

\begin{tabular}{lcc}
\hline $\begin{array}{l}\text { Degree of its end } \\
\text { vertices }(d(u), d(v))\end{array}$ & $\begin{array}{c}\text { Number of edges } \\
\text { (frequency) }\end{array}$ & Ev-degrees \\
\hline$(1,2)$ & $3 n+1$ & 3 \\
$(1,3)(2,2)$ & $13 n+1$ & 4 \\
$(2,3)$ & $29 n-1$ & 5 \\
$(3,3)$ & $11 n-1$ & 6 \\
\hline
\end{tabular}

conjugates with the linear iteration $n$ units. The corresponding molecular graphs of hyaluronic acid-paclitaxel conjugates for $n=1$ and 3 are illustrated in Figure 4. From the molecular structure of HAP, we have $\mid V($ HAP $) \mid=87 n+$ 1 and $\mid E($ HAP $) \mid=96 n$.

As earlier, the edge set of HAP can be divided into twenty-nine edge groups based on ve-degree of its end vertices as summarized in Table 7 .
Now, we establish the expression for the general veinverse sum indeg index for HA-paclitaxel conjugate in the following theorem.

Theorem 4. The general ve-inverse sum indeg index $\operatorname{ISI}_{(\alpha, \beta)}^{v e}$ of HA-paclitaxel conjugate is given by

$$
\begin{aligned}
\operatorname{ISI}_{(\alpha, \beta)}^{\mathrm{ve}(\mathrm{HAP})=} & (n+1)[8]^{\alpha}[6]^{\beta}+6 n[12]^{\alpha}[7]^{\beta}+4 n[18]^{\alpha}[9]^{\beta}+5 n[21]^{\alpha}[10]^{\beta}+n[24]^{\alpha}[11]^{\beta} \\
& +6 n[16]^{\alpha}[8]^{\beta}+6 n[20]^{\alpha}[9]^{\beta}+2 n[24]^{\alpha}[10]^{\beta}+2 n[28]^{\alpha}[11]^{\beta} \\
& +[32]^{\alpha}[12]^{\beta}+2 n[36]^{\alpha}[13]^{\beta}+2 n[40]^{\alpha}[14]^{\beta}+[25]^{\alpha}[10]^{\beta}+n[30]^{\alpha}[11]^{\beta} \\
& +(6 n+2)[35]^{\alpha}[12]^{\beta}+n[40]^{\alpha}[13]^{\beta}+4 n[36]^{\alpha}[12]^{\beta}+(9 n-2)[42]^{\alpha}[13]^{\beta} \\
& +(10 n-2)[48]^{\alpha}[14]^{\beta}+n[54]^{\alpha}[15]^{\beta}+n[60]^{\alpha}[16]^{\beta}+5 n[49]^{\alpha}[14]^{\beta}+4 n[56]^{\alpha}[15]^{\beta} \\
& +4 n[70]^{\alpha}[17]^{\beta}+(4 n-1)[64]^{\alpha}[16]^{\beta}+3 n[80]^{\alpha}[18]^{\beta}+3 n[90]^{\alpha}[19]^{\beta}+n[99]^{\alpha} 20^{\beta} \\
& +2 n[110]^{\alpha}[21]^{\beta} .
\end{aligned}
$$

Proof. Clearly, from Table 4, we have

$$
\begin{aligned}
& \left|E_{2,4}(\mathrm{HAP})\right|=n+1, \\
& \left|E_{3,4}(\mathrm{HAP})\right|=6 n, \\
& \left|E_{3,6}(\mathrm{HAP})\right|=4 n, \\
& \left|E_{3,7}(\mathrm{HAP})\right|=5 n, \\
& \left|E_{3,8}(\mathrm{HAP})\right|=n, \\
& \left|E_{4,4}(\mathrm{HAP})\right|=6 n, \\
& \left|E_{4,5}(\mathrm{HAP})\right|=6 n, \\
& \left|E_{4,6}(\mathrm{HAP})\right|=2 n, \\
& \left|E_{4,7}(\mathrm{HAP})\right|=2 n, \\
& \left|E_{4,8}(\mathrm{HAP})\right|=1, \\
& \left|E_{4,9}(\mathrm{HAP})\right|=2 n, \\
& \left|E_{4,10}(\mathrm{HAP})\right|=2 n, \\
& \left|E_{5,5}(\mathrm{HAP})\right|=1, \\
& \left|E_{5,6}(\mathrm{HAP})\right|=n, \\
& \left|E_{5,7}(\mathrm{HAP})\right|=6 n+2,
\end{aligned}
$$

$$
\begin{aligned}
& \left|E_{5,8}(\mathrm{HAP})\right|=n, \\
& \left|E_{6,6}(\mathrm{HAP})\right|=4 n, \\
& \left|E_{6,7}(\mathrm{HAP})\right|=9 n-2, \\
& \left|E_{6,8}(\mathrm{HAP})\right|=10 n-2, \\
& \left|E_{6,9}(\mathrm{HAP})\right|=n, \\
& \left|E_{6,10}(\mathrm{HAP})\right|=n, \\
& \left|E_{7,7}(\mathrm{HAP})\right|=5 n, \\
& \left|E_{7,8}(\mathrm{HAP})\right|=4 n, \\
& \left|E_{7,10}(\mathrm{HAP})\right|=4 n, \\
& \left|E_{8,8}(\mathrm{HAP})\right|=4 n-1, \\
& \left|E_{8,10}(\mathrm{HAP})\right|=3 n, \\
& \left|E_{9,10}(\mathrm{HAP})\right|=3 n, \\
& \left|E_{9,11}(\mathrm{HAP})\right|=n, \\
& \left|E_{10,11}(\mathrm{HAP})\right|=2 n .
\end{aligned}
$$

Now, applying the definition of the general ve-ISI index, $\operatorname{ISI}_{(\alpha, \beta)}^{\mathrm{ve}}$, we have 


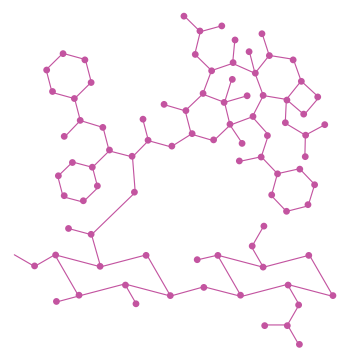

(a)

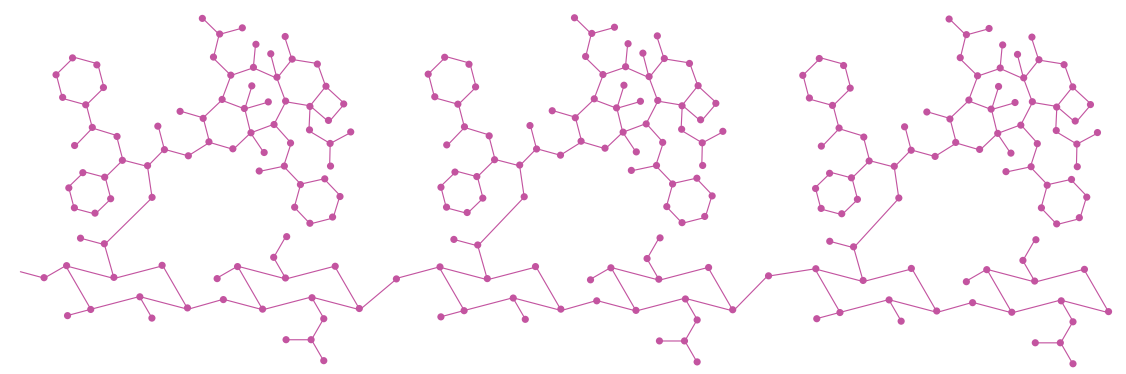

(b)

FIGURE 4: Corresponding molecular graph of hyaluronic acid-paclitaxel conjugates HAP; $n=1, n=3$.

TABLE 7: The ve-degree of the end vertices of edges of HAP.

\begin{tabular}{|c|c|c|}
\hline Ve-degree of its end vertices $\left(d_{\mathrm{ve}}(u), d_{\mathrm{ve}}(v)\right)$ & Number of edges (frequency) & Edge set $E_{i, j}$ \\
\hline$(2,4)$ & $n+1$ & $E_{2,4}$ \\
\hline$(3,4)$ & $6 n$ & $E_{3,4}$ \\
\hline$(3,6)$ & $4 n$ & $E_{3,6}$ \\
\hline$(3,7)$ & $5 n$ & $E_{3,7}$ \\
\hline$(3,8)$ & $N$ & $E_{3,8}$ \\
\hline$(4,4)$ & $6 n$ & $E_{4,4}$ \\
\hline$(4,5)$ & $6 n$ & $E_{4,5}$ \\
\hline$(4,6)$ & $2 n$ & $E_{4,6}$ \\
\hline$(4,7)$ & $2 n$ & $E_{4,7}$ \\
\hline$(4,8)$ & 1 & $E_{4,8}$ \\
\hline$(4,9)$ & $2 n$ & $E_{4,9}$ \\
\hline$(4,10)$ & $2 n$ & $E_{4,10}$ \\
\hline$(5,5)$ & 1 & $E_{5,5}$ \\
\hline$(5,6)$ & $N$ & $E_{5,6}$ \\
\hline$(5,7)$ & $6 n+2$ & $E_{5,7}$ \\
\hline$(5,8)$ & $N$ & $E_{5,8}$ \\
\hline$(6,6)$ & $4 n$ & $E_{6,6}$ \\
\hline$(6,7)$ & $9 n-2$ & $E_{6,7}$ \\
\hline$(6,8)$ & $10 n-2$ & $E_{6,8}$ \\
\hline$(6,9)$ & $N$ & $E_{6,9}$ \\
\hline$(6,10)$ & $N$ & $E_{6,10}$ \\
\hline$(7,7)$ & $5 n$ & $E_{7,7}$ \\
\hline$(7,8)$ & $4 n$ & $E_{7,8}$ \\
\hline$(7,10)$ & $4 n$ & $E_{7,10}$ \\
\hline$(8,8)$ & $4 n-1$ & $E_{8,8}$ \\
\hline$(8,10)$ & $3 n$ & $E_{8,10}$ \\
\hline$(9,10)$ & $3 n$ & $E_{9,10}$ \\
\hline$(9,11)$ & $N$ & $E_{9,11}$ \\
\hline$(10,11)$ & $2 n$ & $E_{10,11}$ \\
\hline
\end{tabular}




$$
\begin{aligned}
& \operatorname{ISI}_{(\alpha, \beta)}^{\mathrm{ve}}(G)=\sum_{u v \in E(G)}\left[d_{\mathrm{ve}}(u) d_{\mathrm{ve}}(v)\right]^{\alpha}\left[d_{\mathrm{ve}}(u)+d_{\mathrm{ve}}(v)\right]^{\beta}, \\
& \operatorname{ISI}_{(\alpha, \beta)}(\mathrm{HAP})=\sum_{u v \in E_{2,4}(\mathrm{HAP})}\left[d_{\mathrm{ve}}(u) d_{\mathrm{ve}}(v)\right]^{\alpha}\left[d_{\mathrm{ve}}(u)+d_{\mathrm{ve}}(v)\right]^{\beta}+\sum_{u v \in E_{3,4}(\mathrm{HAP})}\left[d_{\mathrm{ve}}(u) d_{\mathrm{ve}}(v)\right]^{\alpha}\left[d_{\mathrm{ve}}(u)+d_{\mathrm{ve}}(v)\right]^{\beta}+\ldots \\
& +\sum_{u v \in E_{3,4}(\mathrm{HAP})}\left[d_{\mathrm{ve}}(u) d_{\mathrm{ve}}(v)\right]^{\alpha}\left[d_{\mathrm{ve}}(u)+d_{\mathrm{ve}}(v)\right]^{\beta} \\
& =\left|E_{2,4}(\mathrm{HAP})\right|[(2)(4)]^{\alpha}[2+4]^{\beta}+\left|E_{3,4}(\mathrm{HAP})\right|[(3)(4)]^{\alpha}[3+4]^{\beta}+\left|E_{3,6}(\mathrm{HAP})\right|[(3)(6)]^{\alpha}[3+6]^{\beta} \\
& +\left|E_{3,7}(\mathrm{HAP})\right|[(3)(7)]^{\alpha}[3+7]^{\beta}+\left|E_{3,8}(\mathrm{HAP})\right|[(3)(8)]^{\alpha}[3+8]^{\beta}+\left|E_{4,4}(\mathrm{HAP})\right|[(4)(4)]^{\alpha}[4+4]^{\beta} \\
& +\left|E_{4,5}(\mathrm{HAP})\right|[(4)(5)]^{\alpha}[4+5]^{\beta}+\left|E_{4,6}(\mathrm{HAP})\right|[(4)(6)]^{\alpha}[4+6]^{\beta}+\left|E_{4,7}(\mathrm{HAP})\right|[(4)(7)]^{\alpha}[4+7]^{\beta} \\
& +\left|E_{4,8}(\mathrm{HAP})\right|[(4)(8)]^{\alpha}[4+8]^{\beta}+\left|E_{4,9}(\mathrm{HAP})\right|[(4)(9)]^{\alpha}[4+9]^{\beta}+\left|E_{4,10}(\mathrm{HAP})\right|[(4)(10)]^{\alpha}[4+10]^{\beta} \\
& +\left|E_{5,5}(\mathrm{HAP})\right|[(5)(5)]^{\alpha}[5+5]^{\beta}+\left|E_{5,6}(\mathrm{HAP})\right|[(5)(6)]^{\alpha}[5+6]^{\beta}+\left|E_{5,7}(\mathrm{HAP})\right|[(5)(7)]^{\alpha}[5+7]^{\beta} \\
& +\left|E_{5,8}(\mathrm{HAP})\right|[(5)(8)]^{\alpha}[5+8]^{\beta}+\left|E_{6,6}(\mathrm{HAP})\right|[(6)(6)]^{\alpha}[6+6]^{\beta}+\left|E_{6,7}(\mathrm{HAP})\right|[(6)(7)]^{\alpha}[6+7]^{\beta} \\
& +\left|E_{6,8}(\mathrm{HAP})\right|[(6)(8)]^{\alpha}[6+8]^{\beta}+\left|E_{6,9}(\mathrm{HAP})\right|[(6)(9)]^{\alpha}[6+9]^{\beta}+\left|E_{6,10}(\mathrm{HAP})\right|[(6)(10)]^{\alpha}[6+10]^{\beta} \\
& +\left|E_{7,7}(\mathrm{HAP})\right|[(7)(7)]^{\alpha}[7+7]^{\beta}+\left|E_{7,8}(\mathrm{HAP})\right|[(7)(8)]^{\alpha}[7+8]^{\beta}+\left|E_{7,10}(\mathrm{HAP})\right|[(7)(10)]^{\alpha}[7+10]^{\beta} \\
& +\left|E_{8,8}(\mathrm{HAP})\right|[(8)(8)]^{\alpha}[8+8]^{\beta}+\left|E_{8,10}(\mathrm{HAP})\right|[(8)(10)]^{\alpha}[8+10]^{\beta}+\left|E_{9,10}(\mathrm{HAP})\right|[(9)(10)]^{\alpha}[9+10]^{\beta} \\
& +\left|E_{9,11}(\mathrm{HAP})\right|[(9)(11)]^{\alpha}[9+11]^{\beta}+\left|E_{10,11}(\mathrm{HAP})\right|[(10)(11)]^{\alpha}[10+11]^{\beta} \text {, } \\
& =(n+1)[8]^{\alpha}[6]^{\beta}+6 n[12]^{\alpha}[7]^{\beta}+4 n[18]^{\alpha}[9]^{\beta}+5 n[21]^{\alpha}[10]^{\beta}+n[24]^{\alpha}[11]^{\beta}+6 n[16]^{\alpha}[8]^{\beta} \\
& +6 n[20]^{\alpha}[9]^{\beta}+2 n[24]^{\alpha}[10]^{\beta}+2 n[28]^{\alpha}[11]^{\beta}+[32]^{\alpha}[12]^{\beta}+2 n[36]^{\alpha}[13]^{\beta}+2 n[40]^{\alpha}[14]^{\beta} \\
& +[25]^{\alpha}[10]^{\beta}+n[30]^{\alpha}[11]^{\beta}+(6 n+2)[35]^{\alpha}[12]^{\beta}+n[40]^{\alpha}[13]^{\beta}+4 n[36]^{\alpha}[12]^{\beta}+(9 n-2)[42]^{\alpha}[13]^{\beta} \\
& +(10 n-2)[48]^{\alpha}[14]^{\beta}+n[54]^{\alpha}[15]^{\beta}+n[60]^{\alpha}[16]^{\beta}+5 n[49]^{\alpha}[14]^{\beta}+4 n[56]^{\alpha}[15]^{\beta}+4 n[70]^{\alpha}[17]^{\beta} \\
& +(4 n-1)[64]^{\alpha}[16]^{\beta}+3 n[80]^{\alpha}[18]^{\beta}+3 n[90]^{\alpha}[19]^{\beta}+n[99]^{\alpha} 20^{\beta}+2 n[110]^{\alpha}[21]^{\beta} \text {. }
\end{aligned}
$$

Hence, the theorem.

Next, we give exact values of the most well-known vedegree-based indices of HA-paclitaxel conjugate. (i) $M_{1}^{\beta v e}(H A P)=1210 n-18$

(ii) $M_{2}^{v e}(H A P)=3983 n-109$

(iii)

Corollary 4. Let HAP be the molecular graph of HA-paclitaxel conjugate; then,

$$
\begin{aligned}
R^{\mathrm{ve}}(\mathrm{HAP})= & \left(\frac{11}{6 \sqrt{2}}+\frac{11}{2 \sqrt{3}}+\frac{15}{4 \sqrt{5}}+\frac{11}{6 \sqrt{6}}+\frac{1}{\sqrt{7}}+\frac{5}{\sqrt{10}}+\frac{1}{3 \sqrt{11}}+\frac{2}{\sqrt{14}}+\frac{1}{2 \sqrt{15}}+\frac{5}{\sqrt{21}}+\frac{1}{\sqrt{30}}+\frac{6}{\sqrt{35}}+\frac{9}{\sqrt{42}}+\frac{4}{\sqrt{70}}+\frac{2}{\sqrt{110}}+\frac{26}{7}\right) n \\
& +\left(\frac{3}{4 \sqrt{2}}-\frac{2}{4 \sqrt{3}}+\frac{2}{\sqrt{35}}-\frac{2}{\sqrt{42}}+\frac{3}{40}\right) .
\end{aligned}
$$

(iv)

$$
\begin{aligned}
\chi^{\text {ve }}(\text { HAP })= & \left(\frac{4}{\sqrt{2}}+\frac{10}{2 \sqrt{3}}+\frac{3}{2 \sqrt{5}}+\frac{1}{\sqrt{6}}+\frac{6}{\sqrt{7}}+\frac{7}{\sqrt{10}}+\frac{4}{\sqrt{11}}+\frac{12}{\sqrt{13}}+\frac{17}{\sqrt{14}}+\frac{5}{\sqrt{15}}+\frac{4}{\sqrt{17}}+\frac{3}{\sqrt{19}}+\frac{2}{\sqrt{21}}+\frac{55}{12}\right) n \\
& +\left(\frac{3}{2 \sqrt{2}}+\frac{1}{\sqrt{6}}+\frac{1}{\sqrt{10}}-\frac{2}{\sqrt{13}}-\frac{2}{\sqrt{14}}-\frac{1}{4}\right) .
\end{aligned}
$$


(v)

$$
H^{\mathrm{ve}}(\mathrm{HAP})=\frac{1925236355 n+36656301}{232792560} .
$$

(vi) $H M_{1}^{v e}(H A P)=16406 n-418$ (vii) $H_{2}^{v e}(H A P)=215953 n-8069$

(viii) $\operatorname{ReZG}_{3}^{v e}(H A P)=57712 n-1938$

(ix)

$\mathrm{GA}^{\mathrm{ve}}(\mathrm{HAP})=\left(\frac{\sqrt{35}+\sqrt{21}+5}{2}+\frac{5 \sqrt{2}+6 \sqrt{5}}{3}+\frac{7 \sqrt{6}}{5}+\frac{32 \sqrt{3}+2 \sqrt{10}}{7}+\frac{\sqrt{15}}{8}+\frac{2 \sqrt{6}+4 \sqrt{7}+\sqrt{30}}{11}+\frac{2 \sqrt{10}+9 \sqrt{42}+12}{13}\right.$

$$
\left.+\frac{4 \sqrt{70}}{17}+\frac{3 \sqrt{10}}{19}+\frac{3 \sqrt{11}}{20}+\frac{2 \sqrt{110}}{21}+7\right) n+\frac{2 \sqrt{2}}{3}+\frac{\sqrt{35}}{6}-\frac{2 \sqrt{42}}{13}-\frac{4 \sqrt{3}}{7} .
$$

(x)

$$
\begin{aligned}
\mathrm{AG}^{\mathrm{ve}}(\mathrm{HAP})= & \left(\frac{15}{\sqrt{2}}+\frac{56}{\sqrt{3}}+\frac{81}{2 \sqrt{5}}+\frac{41}{2 \sqrt{6}}+\frac{11}{\sqrt{7}}+\frac{79}{2 \sqrt{10}}+\frac{20}{3 \sqrt{11}}+\frac{30}{\sqrt{14}}+\frac{8}{\sqrt{15}}+\frac{50}{\sqrt{21}}+\frac{11}{\sqrt{30}}+\frac{72}{\sqrt{35}}+\frac{117}{\sqrt{42}}+\frac{68}{\sqrt{70}}+\frac{42}{\sqrt{110}}+\frac{127}{3}\right) n \\
& +\left(\frac{6}{\sqrt{2}}+\frac{7}{\sqrt{3}}+\frac{24}{\sqrt{35}}-\frac{26}{\sqrt{42}}\right) .
\end{aligned}
$$

$(x i)$

Proof. From Theorem 4, we have

$\operatorname{ISI}^{\mathrm{ve}}(\mathrm{HAP})=\operatorname{ISI}^{\mathrm{ve}}(\mathrm{HAP})=74 n+\frac{385639676 n-1737740}{1763580}-4$.

$$
\begin{aligned}
\operatorname{ISI}_{(\alpha, \beta)}^{\mathrm{ve}(\mathrm{HAP})=} & (n+1)[8]^{\alpha}[6]^{\beta}+6 n[12]^{\alpha}[7]^{\beta}+4 n[18]^{\alpha}[9]^{\beta}+5 n[21]^{\alpha}[10]^{\beta}+n[24]^{\alpha}[11]^{\beta} \\
& +6 n[16]^{\alpha}[8]^{\beta}+6 n[20]^{\alpha}[9]^{\beta}+2 n[24]^{\alpha}[10]^{\beta}+2 n[28]^{\alpha}[11]^{\beta}+[32]^{\alpha}[12]^{\beta}+2 n[36]^{\alpha}[13]^{\beta} \\
& +2 n[40]^{\alpha}[14]^{\beta}+[25]^{\alpha}[10]^{\beta}+n[30]^{\alpha}[11]^{\beta}+(6 n+2)[35]^{\alpha}[12]^{\beta}+n[40]^{\alpha}[13]^{\beta}+4 n[36]^{\alpha}[12]^{\beta} \\
& +(9 n-2)[42]^{\alpha}[13]^{\beta}+(10 n-2)[48]^{\alpha}[14]^{\beta}+n[54]^{\alpha}[15]^{\beta}+n[60]^{\alpha}[16]^{\beta}+5 n[49]^{\alpha}[14]^{\beta} \\
& +4 n[56]^{\alpha}[15]^{\beta}+4 n[70]^{\alpha}[17]^{\beta}+(4 n-1)[64]^{\alpha}[16]^{\beta}+3 n[80]^{\alpha}[18]^{\beta}+3 n[90]^{\alpha}[19]^{\beta}+n[99]^{\alpha}[20]^{\beta} \\
& +2 n[110]^{\alpha}[21]^{\beta} .
\end{aligned}
$$

(i) Substitute $\alpha=0$ and $\beta=1$ in equation (56); then, the first ve-degree Zagreb beta index is

$$
\operatorname{ISI}_{(0,1)}^{\mathrm{ve}}(\mathrm{HAP})=M_{1}^{\beta \mathrm{ve}}(\mathrm{HAP})=1210 n-18 .
$$

(ii) If $\alpha=1$ and $\beta=0$ are chosen, then $\operatorname{ISI}_{(1,0)}^{\mathrm{ve}}(\mathrm{HAP})=$ $M_{2}^{\mathrm{ve}}$ (HAP) is the second ve-degree Zagreb index given by

$$
M_{2}^{\beta \mathrm{ve}}(G)=3983 n-109 .
$$


(iii) For $\alpha=-(1 / 2)$ and $\beta=0$, equation (56) gives the ve-degree Randić index, i.e.,

$$
\begin{aligned}
\operatorname{ISI}_{(-(1 / 2), 0)}^{\mathrm{ve}}(\mathrm{HAP})= & R^{\mathrm{ve}}(\mathrm{HAP})=\left(\frac{11}{6 \sqrt{2}}+\frac{11}{2 \sqrt{3}}+\frac{15}{4 \sqrt{5}}+\frac{11}{6 \sqrt{6}}+\frac{1}{\sqrt{7}}+\frac{5}{\sqrt{10}}+\frac{1}{3 \sqrt{11}}+\frac{2}{\sqrt{14}}+\frac{1}{2 \sqrt{15}}+\frac{5}{\sqrt{21}}+\frac{1}{\sqrt{30}}\right. \\
& \left.+\frac{6}{\sqrt{35}}+\frac{9}{\sqrt{42}}+\frac{4}{\sqrt{70}}+\frac{2}{\sqrt{110}}+\frac{26}{7}\right) n+\left(\frac{3}{4 \sqrt{2}}-\frac{2}{4 \sqrt{3}}+\frac{2}{\sqrt{35}}-\frac{2}{\sqrt{42}}+\frac{3}{40}\right) .
\end{aligned}
$$

(iv) If $\alpha=0$ and $\beta=-(1 / 2)$ are chosen, then $\operatorname{ISI}_{(0,-(1 / 2))}^{\mathrm{ve}}(\mathrm{HAP})=\chi^{\mathrm{ve}}(\mathrm{HAP})$, and we obtain the ve-degree sum-connectivity index as

$$
\begin{aligned}
\chi^{\mathrm{ve}}(\text { HAP })= & \left(\frac{4}{\sqrt{2}}+\frac{10}{2 \sqrt{3}}+\frac{3}{2 \sqrt{5}}+\frac{1}{\sqrt{6}}+\frac{6}{\sqrt{7}}+\frac{7}{\sqrt{10}}+\frac{4}{\sqrt{11}}+\frac{12}{\sqrt{13}}+\frac{17}{\sqrt{14}}+\frac{5}{\sqrt{15}}+\frac{4}{\sqrt{17}}+\frac{3}{\sqrt{19}}+\frac{2}{\sqrt{21}}+\frac{55}{12}\right) n \\
& +\left(\frac{3}{2 \sqrt{2}}+\frac{1}{\sqrt{6}}+\frac{1}{\sqrt{10}}-\frac{2}{\sqrt{13}}-\frac{2}{\sqrt{14}}-\frac{1}{4}\right) .
\end{aligned}
$$

(v) Substitute $\alpha=0$ and $\beta=-1$ in equation (56) and then multiply this equation by 2 ; we get the vedegree harmonic index as

$\operatorname{ISI}_{(0,-1)}^{\mathrm{ve}}($ HAP $)=H^{\mathrm{ve}}($ HAP $)=\frac{1925236355 n+36656301}{232792560}$.

(vi) If $\alpha=0$ and $\beta=2$, then the first hyper ve-degree Zagreb index

$$
\operatorname{ISI}_{(0,2)}^{\mathrm{ve}}(\mathrm{HAP})=\mathrm{HM}_{1}^{\mathrm{ve}}(\mathrm{HAP})=16406 n-418 \text {. }
$$

(vii) Substituting $\alpha=2$ and $\beta=0$, we get

$$
\operatorname{ISI}_{(2,0)}^{\mathrm{ve}}(\mathrm{HAP})=\mathrm{HM}_{2}^{\mathrm{ve}}(\mathrm{HAP})=215953 n-8069 .
$$

(viii) If $\alpha=1$ and $\beta=1$, then we have the redefined third ve-degree Zagreb index,

$$
\operatorname{ISI}_{(1,1)}^{\mathrm{ve}}(\mathrm{HAP})=\operatorname{ReZG}_{3}^{\mathrm{ve}}(\mathrm{HAP})=57712 n-1938 .
$$

(ix) If we take $\alpha=1 / 2$ and $\beta=-1$ in equation (56) and then multiply the result by 2 , this gives the vedegree geometric-arithmetic index $\mathrm{GA}^{\mathrm{ve}}(\mathrm{HAP})$ as

$$
\begin{aligned}
\mathrm{GA}^{\mathrm{ve}}(\mathrm{HAP})= & \left(\frac{\sqrt{35}+\sqrt{21}+5}{2}+\frac{5 \sqrt{2}+6 \sqrt{5}}{3}+\frac{7 \sqrt{6}}{5}+\frac{32 \sqrt{3}+2 \sqrt{10}}{7}+\frac{\sqrt{15}}{8}+\frac{2 \sqrt{6}+4 \sqrt{7}+\sqrt{30}}{11}\right. \\
& \left.+\frac{2 \sqrt{10}+9 \sqrt{42}+12}{13}+\frac{4 \sqrt{70}}{17}+\frac{3 \sqrt{10}}{19}+\frac{3 \sqrt{11}}{20}+\frac{2 \sqrt{110}}{21}+7\right) n+\left(\frac{2 \sqrt{2}}{3}+\frac{\sqrt{35}}{6}-\frac{2 \sqrt{42}}{13}-\frac{4 \sqrt{3}}{7}\right) .
\end{aligned}
$$


(x) By taking $\alpha=-(1 / 2)$ and $\beta=1$ in equation (56) and multiplying by $1 / 2$, we get

$$
\begin{aligned}
\mathrm{AG}^{\mathrm{ve}}(\mathrm{HAP})= & \left(\frac{15}{\sqrt{2}}+\frac{56}{\sqrt{3}}+\frac{81}{2 \sqrt{5}}+\frac{41}{2 \sqrt{6}}+\frac{11}{\sqrt{7}}+\frac{79}{2 \sqrt{10}}+\frac{20}{3 \sqrt{11}}+\frac{30}{\sqrt{14}}+\frac{8}{\sqrt{15}}+\frac{50}{\sqrt{21}}+\frac{11}{\sqrt{30}}+\frac{72}{\sqrt{35}}\right. \\
& \left.+\frac{117}{\sqrt{42}}+\frac{68}{\sqrt{70}}+\frac{42}{\sqrt{110}}+\frac{127}{3}\right) n+\left(\frac{6}{\sqrt{2}}+\frac{7}{\sqrt{3}}+\frac{24}{\sqrt{35}}-\frac{26}{\sqrt{42}}\right) .
\end{aligned}
$$

(xi) If $\alpha=1$ and $\beta=-1$, then we have the ve-degree inverse sum indeg index

$$
\mathrm{ISI}^{\mathrm{ve}}(\mathrm{HAP})=74 n+\frac{385639676 n-1737740}{1763580}-4 .
$$

Next, we summarized vertex set partition of HAP based on its ve-degree as given in Table 8 .

Theorem 5. The general ve-Zagreb index $M_{\alpha}^{v e}$ of $H A$ paclitaxel

$$
\begin{aligned}
M_{\alpha}^{\mathrm{ve}}(\mathrm{HAP})= & (n+1)[2]^{\alpha}+16 n[3]^{\alpha}+(17 n+1)[4]^{\alpha} \\
& +(7 n+2)[5]^{\alpha}+(16 n-2)[6]^{\alpha}+14 n[7]^{\alpha} \\
& +(9 n-1)[8]^{\alpha}+2 n[9]^{\alpha} \\
& +4 n[10]^{\alpha}+n[11]^{\alpha} .
\end{aligned}
$$

Proof. From Table 8, we have

$$
\begin{aligned}
& \left|V_{2}(\mathrm{HAP})\right|=n+1, \\
& \left|V_{3}(\mathrm{HAP})\right|=16 n, \\
& \left|V_{4}(\mathrm{HAP})\right|=17 n+1, \\
& \left|V_{5}(\mathrm{HAP})\right|=7 n+2, \\
& \left|V_{6}(\mathrm{HAP})\right|=16 n-2, \\
& \left|V_{7}(\mathrm{HAP})\right|=14 n, \\
& \left|V_{8}(\mathrm{HAP})\right|=9 n-1, \\
& \left|V_{9}(\mathrm{HAP})\right|=2 n, \\
& \left|V_{10}(\mathrm{HAP})\right|=4 n, \\
& \left|V_{11}(\mathrm{HAP})\right|=n .
\end{aligned}
$$
have

By the definition of the general ve-Zagreb index $M_{\alpha}^{\mathrm{ve}}$, we

$$
\begin{aligned}
M_{\alpha}^{\mathrm{ve}}(G)= & \sum_{u \in V(G)}\left[d_{\mathrm{ve}}(u)\right]^{\alpha}, \\
M_{\alpha}^{\mathrm{ve}}(\mathrm{HAP})= & \sum_{u \in V_{2}(\mathrm{HAP})}\left[d_{\mathrm{ve}}(u)\right]^{\alpha}+\sum_{u \in V_{3}(\mathrm{HAP})}\left[d_{\mathrm{ve}}(u)\right]^{\alpha}+\sum_{u \in V_{4}(\mathrm{HAP})}\left[d_{\mathrm{ve}}(u)\right]^{\alpha} \\
& +\sum_{u \in V_{5}(\mathrm{HAP})}\left[d_{\mathrm{ve}}(u)\right]^{\alpha}+\sum_{u \in V_{6}(\mathrm{HAP})}\left[d_{\mathrm{ve}}(u)\right]^{\alpha}+\sum_{u \in V_{7}(\mathrm{HAP})}\left[d_{\mathrm{ve}}(u)\right]^{\alpha} \\
& +\sum_{u \in V_{8}(\mathrm{HAP})}\left[d_{\mathrm{ve}}(u)\right]^{\alpha}+\sum_{u \in V_{9}(\mathrm{HAP})}\left[d_{\mathrm{ve}}(u)\right]^{\alpha}+\sum_{u \in V_{10}(\mathrm{HAP})}\left[d_{\mathrm{ve}}(u)\right]^{\alpha}+\sum_{u \in V_{11}(\mathrm{HAP})}\left[d_{\mathrm{ve}}(u)\right]^{\alpha}, \\
M_{\alpha}^{\mathrm{ve}}(\mathrm{HAP})= & V_{2}(\mathrm{HAP})\left|[2]^{\alpha}+\right| V_{3}(\mathrm{HAP})\left|[3]^{\alpha}+\right| V_{4}(\mathrm{HAP})\left|[4]^{\alpha}+\right| V_{5}(\mathrm{HAP})\left|[5]^{\alpha}+\right| V_{6}(\mathrm{HAP})\left|[6]^{\alpha}+\right| V_{7}(\mathrm{HAP}) \mid[7]^{\alpha} \\
& +\left|V_{8}(\mathrm{HAP})\right|[8]^{\alpha}+\left|V_{9}(\mathrm{HAP})\right|[9]^{\alpha}+\left|V_{10}(\mathrm{HAP})\right|[10]^{\alpha}+\left|V_{11}(\mathrm{HAP})\right|[11]^{\alpha}, \\
M_{\alpha}^{\mathrm{ve}}(\mathrm{HAP})= & (n+1)[2]^{\alpha}+16 n[3]^{\alpha}+(17 n+1)[4]^{\alpha}+(7 n+2)[5]^{\alpha}+(16 n-2)[6]^{\alpha}+14 n[7]^{\alpha}+(9 n-1)[8]^{\alpha}+2 n[9]^{\alpha} \\
& +4 n[10]^{\alpha}+n[11]^{\alpha} .
\end{aligned}
$$

Using Theorem 5, the following corollary can be obtained with little efforts.

Corollary 5. Let HAP be the molecular graph of HA-paclitaxel conjugate; then,
(i) $T^{v e}(H A P)=488 n-4$
(ii) $M_{1}^{\alpha v e}(H A P)=3116 n-66$
(iii) $F_{1}^{\text {ve }}(H A P)=22058 n-622$
(iv) $I D^{v e}(H A P)=(63313 n+2739) / 3960$ 
TABLE 8: The ve-degree of the vertices of HAP.

\begin{tabular}{lcc}
\hline Ve-degree $d_{\mathrm{ve}}(u)$ & Number of vertices (frequency) & Vertex set $V_{i}$ \\
\hline 2 & $n+1$ & $V_{2}$ \\
3 & $16 n$ & $V_{3}$ \\
4 & $17 n+1$ & $V_{4}$ \\
5 & $7 n+2$ & $V_{5}$ \\
6 & $16 n-2$ & $V_{6}$ \\
7 & $14 n$ & $V_{7}$ \\
8 & $9 n-1$ & $V_{8}$ \\
9 & $2 n$ & $V_{9}$ \\
10 & $4 n$ & $V_{10}$ \\
11 & $N$ & $V_{11}$ \\
\hline
\end{tabular}

$(v)$

$$
\mathrm{ZD}^{\mathrm{ve}}(\mathrm{HAP})=\left(\frac{1}{\sqrt{2}}+\frac{16}{\sqrt{3}}+\frac{7}{\sqrt{5}}+\frac{16}{\sqrt{6}}+\frac{14}{\sqrt{7}}+\frac{9}{\sqrt{8}}+\frac{4}{\sqrt{10}}+\frac{1}{\sqrt{11}}+\frac{55}{6}\right) n+\left(\frac{1}{\sqrt{2}}+\frac{2}{\sqrt{5}}-\frac{2}{\sqrt{6}}-\frac{1}{2 \sqrt{2}}+\frac{1}{2}\right)
$$

(vi) $m M_{1}^{v e}(H A P)=(473554859 n+35271621)$ $/ 109771200$

Proof. From Theorem 5, we have

$$
\begin{aligned}
M_{\alpha}^{\mathrm{ve}}(\mathrm{HAP})= & (n+1)[2]^{\alpha}+16 n[3]^{\alpha}+(17 n+1)[4]^{\alpha} \\
& +(7 n+2)[5]^{\alpha}+(16 n-2)[6]^{\alpha}+14 n[7]^{\alpha} \\
& +(9 n-1)[8]^{\alpha}+2 n[9]^{\alpha} \\
& +4 n[10]^{\alpha}+n[11]^{\alpha} .
\end{aligned}
$$

(i) Substituting $\quad \alpha=1$ in equation (72), give $T^{\mathrm{ve}}(\mathrm{HAP})=488 n-4$ (ii) Substitute $\alpha=2$ in equation (72); then, $M_{2}^{\mathrm{ve}}(\mathrm{HAC})=M_{1}^{\alpha \mathrm{ve}}(\mathrm{HAC})=3116 n-66$ is the first ve-degree Zagreb alpha index.

(iii) Substituting $\alpha=3$ in equation (72) gives the $F_{1}$-vedegree index, i.e.,

$M_{3}^{\mathrm{ve}}(\mathrm{HAC})=F_{1}^{\mathrm{ve}}(\mathrm{HAC})=22058 n-622$.

(iv) For $\alpha=-1$, equation (72) gives the inverse ve-degree index, i.e.,

$$
M_{-1}^{\mathrm{ve}}(\mathrm{HAC})=\operatorname{ID}^{\mathrm{ve}}(G)=\frac{63313 n+2739}{3960} .
$$

(v) If $\alpha=-(1 / 2)$, then $M_{-(1 / 2)}^{\mathrm{ve}}(\mathrm{HAC})=\mathrm{ZD}^{\mathrm{ve}}(G)$ zeroth order ve-degree index and

$$
\mathrm{ZD}^{\mathrm{ve}}(\mathrm{HAP})=\left(\frac{1}{\sqrt{2}}+\frac{16}{\sqrt{3}}+\frac{7}{\sqrt{5}}+\frac{16}{\sqrt{6}}+\frac{14}{\sqrt{7}}+\frac{9}{\sqrt{8}}+\frac{4}{\sqrt{10}}+\frac{1}{\sqrt{11}}+\frac{55}{6}\right) n+\left(\frac{1}{\sqrt{2}}+\frac{2}{\sqrt{5}}-\frac{2}{\sqrt{6}}-\frac{1}{2 \sqrt{2}}+\frac{1}{2}\right)
$$

(vi) If we put $\alpha=-2$ in equation (72), we get the modified first ve-degree index, i.e.,

$$
M_{-2}^{\mathrm{ve}}(\mathrm{HAC})=\mathrm{mM}_{1}^{\mathrm{ve}}(G)=\frac{473554859 n+35271621}{109771200} .
$$

Finally, we have a partition of the edge set of HAP on the basis of ev-degree of edges, as given in Table 9.

Theorem 6. The general ev-degree index $M_{\alpha}^{e v}$ of HA-paclitaxel conjugate is given by

$$
\begin{aligned}
M_{\alpha}^{\mathrm{ev}}(\mathrm{HAP})= & (n+1)[3]^{\alpha}+(29 n+1)[4]^{\alpha} \\
& +(36 n-1)[5]^{\alpha}+(22 n-1)[6]^{\alpha} \\
& +7 n[7]^{\alpha}+n[8]^{\alpha}
\end{aligned}
$$

Proof. Clearly,

$$
\begin{aligned}
& \left|E_{3}(\mathrm{HAP})\right|=n+1, \\
& \left|E_{4}(\mathrm{HAP})\right|=29 n+1, \\
& \left|E_{5}(\mathrm{HAP})\right|=36 n-1, \\
& \left|E_{6}(\mathrm{HAP})\right|=22 n-1, \\
& \left|E_{7}(\mathrm{HAP})\right|=7 n, \\
& \left|E_{8}(\mathrm{HAP})\right|=n .
\end{aligned}
$$


TABLE 9: The ev-degree of the edges of HAP.

\begin{tabular}{lcc}
\hline Degree of its end vertices $(d(u), d(v))$ & Number of edges (frequency) & Ev-degrees \\
\hline$(1,2)$ & $n+1$ & 3 \\
$(1,3)(2,2)$ & $29 n+1$ & 4 \\
$(1,4)(2,3)$ & $36 n-1$ & 5 \\
$(2,4)(3,3)$ & $22 n-1$ & 6 \\
$(3,4)$ & $7 n$ & 7 \\
$(4,4)$ & $n$ & 8 \\
\hline
\end{tabular}
have

Applying the definition of the ev-degree index $M_{\alpha}^{\mathrm{ev}}$, we

$$
\begin{aligned}
M_{\alpha}^{\mathrm{ev}}(G)= & \sum_{e \in E(G)}\left[d_{\mathrm{ev}}(e)\right]^{\alpha}, \\
M_{\alpha}^{\mathrm{ev}}(\mathrm{HAP})= & \sum_{e \in E_{3}(\mathrm{HAP})}\left[d_{\mathrm{ev}}(e)\right]^{\alpha}+\sum_{e \in E_{4}(\mathrm{HAP})}\left[d_{\mathrm{ev}}(e)\right]^{\alpha}+\sum_{e \in E_{5}(\mathrm{HAP})}\left[d_{\mathrm{ev}}(e)\right]^{\alpha} \\
& +\sum_{e \in E_{6}(\mathrm{HAP})}\left[d_{\mathrm{ev}}(e)\right]^{\alpha}+\sum_{e \in E_{7}(\mathrm{HAP})}\left[d_{\mathrm{ev}}(e)\right]^{\alpha}+\sum_{e \in E_{8}(\mathrm{HAP})}\left[d_{\mathrm{ev}}(e)\right]^{\alpha}, \\
M_{\alpha}^{\mathrm{ev}}(\mathrm{HAP})= & \left|E_{3}(\mathrm{HAP})\right|[3]^{\alpha}+\left|E_{4}(\mathrm{HAP})\right|[4]^{\alpha}+\left|E_{5}(\mathrm{HAP})\right|[5]^{\alpha}+\left|E_{6}(\mathrm{HAP})\right|[6]^{\alpha}+\left|E_{7}(\mathrm{HAP})\right|[7]^{\alpha}+\left|E_{8}(\mathrm{HAP})\right|[8]^{\alpha}, \\
M_{\alpha}^{\mathrm{ev}}(\mathrm{HAP})= & (n+1)[3]^{\alpha}+(29 n+1)[4]^{\alpha}+(36 n-1)[5]^{\alpha}+(22 n-1)[6]^{\alpha}+7 n[7]^{\alpha}+n[8]^{\alpha} .
\end{aligned}
$$

Next, corollary is immediate from Theorem 6.

Corollary 6. Let HAP be the molecular graph of HA-paclitaxel conjugate; then,

(i) $T^{e v}(H A P)=488 n-4$

(ii) $M^{\text {ev }}(H A P)=2572 n-36$ (iii) $F^{e v}(H A P)=14048 n-250$

(iv) $m M^{e v}(H A P)=(416627 n+10668) / 100800$

(v) $I D^{e v}(H A P)=n+((2229 n+26) / 120)$

(vi)

$$
R^{\mathrm{ev}}(\mathrm{HAP})=\left(\frac{1}{\sqrt{3}}+\frac{36}{\sqrt{5}}+\frac{22}{\sqrt{6}}+\frac{7}{\sqrt{7}}+\frac{1}{\sqrt{8}}+\frac{29}{2}\right) n+\left(\frac{1}{\sqrt{3}}-\frac{1}{\sqrt{5}}-\frac{1}{\sqrt{6}}+\frac{1}{2}\right)
$$

(vii) $R^{\text {ev }}(H A P)=(\sqrt{3}+36 \sqrt{5}+22 \sqrt{6}+7 \sqrt{7}+$

$\sqrt{8}+58) n+(\sqrt{3}-\sqrt{5}-\sqrt{6}+2)$

Proof. Substituting $\alpha=1,2,3,-2,-1,-(1 / 2),(1 / 2)$ in the expression of $M_{\alpha}^{\mathrm{ev}}$ (HAP) from Theorem 6, we get desired results.

\section{Chemical Significance of the Newly Introduced Indices}

In this section, we compute $\operatorname{ISI}_{(\alpha, \beta)}^{v e}(G), \operatorname{ISI}_{(\alpha, \beta)}^{v e}(G)$ $(\alpha, \beta)=(2,0),(0,2), M_{\alpha}^{v e}(G), M_{\alpha}^{e v}(G)$ for $\alpha=3$ of octane isomers as summarized in Table 10 and investigate the predictive power of these indices for certain physicochemical properties such as entropy $(S)$, acentric factor (AcenFac), enthalpy of vaporization (HVAP), and standard enthalpy of vaporization (DHVAP) of octane isomers available at http://www.moleculardescriptors.eu (Table 11). Next, we model selected physicochemical properties of octane isomers with $\operatorname{ISI}_{(0,2)}^{v e}(G), \operatorname{ISI}_{(2,0)}^{v e}(G) M_{3}^{v e}(G)$, and $M_{3}^{\text {ev }}(G)$ (Table 12).

It can be easily seen from Table 12 that the most convenient indices which are modeling the entropy $(S)$, enthalpy of vaporization (HVAP), standard enthalpy of vaporization (DHVAP), and acentric factor (AcenFac) are as follows:

(i) $\operatorname{ISI}_{(0,2)}^{v e}(G)$ for the acentric factor

(ii) $M_{3}^{v e}(G)$ for enthalpy of vaporization (HVAP) and standard enthalpy of vaporization (DHVAP)

(iii) $M_{3}^{e v}(G)$ for entropy

Graphical representation of these correlations is shown in Figure 5.

Note that all indices show the negative strong correlation; because of this fact, these graph invariants are compared with each other by using the squares of correlation coefficients to ensure the compliance (conformity) between the positive and negative correlation coefficients (Table 13). Furthermore, it can be seen from Tables 12 and 13 that all the indices have a good 
TABLE 10: Various ve-degree and ev-degree topological indices of octane isomers.

\begin{tabular}{|c|c|c|c|c|}
\hline Molecule & $\operatorname{ISI}_{(0,2)}^{v e}(G)$ & $\operatorname{ISI}_{(2,0)}^{v e}(G)$ & $M_{3}^{v e}(G)$ & $M_{3}^{e v}(G)$ \\
\hline n-Octane & 340 & 1128 & 326 & 374 \\
\hline 2-Methyl-heptane & 398 & 1524 & 406 & 472 \\
\hline 3-Methyl-heptane & 400 & 1525 & 387 & 496 \\
\hline 4-Methyl-heptane & 442 & 1997 & 472 & 496 \\
\hline 2,3-Dimethyl-hexane & 540 & 2835 & 582 & 624 \\
\hline 2,4-Dimethyl-hexane & 500 & 2453 & 558 & 594 \\
\hline 2,5-Dimethyl-hexane & 458 & 2001 & 486 & 570 \\
\hline 3,4-Dimethyl-hexane & 578 & 3224 & 630 & 648 \\
\hline 2,2-Dimethyl-hexane & 538 & 2856 & 632 & 746 \\
\hline 3,3-Dimethyl-hexane & 620 & 3808 & 728 & 800 \\
\hline 3-Ethyl-hexane & 482 & 2441 & 520 & 520 \\
\hline 2,2,3-Trimethyl-pentane & 726 & 4781 & 850 & 934 \\
\hline 2,2,4-Trimethyl-pentane & 688 & 4564 & 869 & 844 \\
\hline 2,3,3-Trimethyl-pentane & 766 & 5305 & 874 & 964 \\
\hline 2,3,4-Trimethyl-pentane & 644 & 3791 & 728 & 752 \\
\hline 2-Methyl-3-ethyl-pentane & 586 & 3371 & 666 & 648 \\
\hline 3-Methyl-3-ethyl-pentane & 700 & 4759 & 806 & 854 \\
\hline 2,2,3,3-Tetramethylbutane & 922 & 7105 & 1070 & 1262 \\
\hline
\end{tabular}

TABLE 11: Some physicochemical properties of octane isomers.

\begin{tabular}{|c|c|c|c|c|}
\hline Molecule & Entropy $(S)$ & HVAP & DHVAP & AcenFac \\
\hline n-Octane & 111.67 & 73.19 & 9.915 & 0.397898 \\
\hline 2-Methyl-heptane & 109.84 & 70.3 & 9.484 & 0.377916 \\
\hline 3-Methyl-heptane & 111.26 & 71.3 & 9.521 & 0.371002 \\
\hline 4-Methyl-heptane & 109.32 & 70.91 & 9.483 & 0.371504 \\
\hline 2,3-Dimethyl-hexane & 108.02 & 70.2 & 9.272 & 0.348247 \\
\hline 2,4-Dimethyl-hexane & 106.98 & 68.5 & 9.029 & 0.344223 \\
\hline 2,5-Dimethyl-hexane & 105.72 & 68.6 & 9.051 & 0.35683 \\
\hline 3,4-Dimethyl-hexane & 106.59 & 70.2 & 9.316 & 0.340345 \\
\hline 2,2-Dimethyl-hexane & 103.42 & 67.7 & 8.915 & 0.339426 \\
\hline 3,3-Dimethyl-hexane & 104.74 & 68.5 & 8.973 & 0.322596 \\
\hline 3-Ethyl-hexane & 109.43 & 71.7 & 9.476 & 0.362472 \\
\hline 2,2,3-Trimethyl-pentane & 101.31 & 67.3 & 8.826 & 0.300816 \\
\hline 2,2,4-Trimethyl-pentane & 104.09 & 64.87 & 8.402 & 0.30537 \\
\hline 2,3,3-Trimethyl-pentane & 102.06 & 68.1 & 8.897 & 0.293177 \\
\hline 2,3,4-Trimethyl-pentane & 102.39 & 68.37 & 9.014 & 0.317422 \\
\hline 2-Methyl-3-ethyl-pentane & 106.06 & 69.7 & 9.209 & 0.332433 \\
\hline 3-Methyl-3-ethyl-pentane & 101.48 & 69.3 & 9.081 & 0.306899 \\
\hline 2,2,3,3-Tetramethylbutane & 93.06 & 66.2 & 8.41 & 0.255294 \\
\hline
\end{tabular}

TABLE 12: The correlation between ve-degree and ev-degree topological indices and many physicochemical properties of octane isomers.

\begin{tabular}{lcccc}
\hline Index & Entropy $(S)$ & HVAP & DHVAP & AcenFac \\
\hline $\operatorname{ISI}_{(0,2)}^{v e}(G)$ & -0.93637344 & -0.759290239 & -0.838405607 & $-\mathbf{0 . 9 9 1 6 5 1 3 2}$ \\
$\operatorname{ISI}_{(2,0)}^{v e}(G)$ & -0.93263275 & -0.740521517 & -0.824163005 & -0.9848198 \\
$M_{3}^{v e}(G)$ & -0.92648728 & $-\mathbf{0 . 8 1 3 1 3 2 5 2 3}$ & $\mathbf{- 0 . 8 8 0 0 5 1 4 0 6}$ & -0.98993655 \\
$M_{3}^{\text {ev }}(G)$ & $-\mathbf{0 . 9 6 2 3 9 6 7 6 4}$ & -0.795535677 & -0.867170763 & -0.981777498 \\
\hline
\end{tabular}

Bold values show highest values of correlation between topological indices and properties.

correlation with entropy $(S)$ and have a strong correlation with the acentric factor. But none of the indices are able to model enthalpy of vaporization (HVAP) strongly.
For the above reasons, it can be argued that these vedegree and ev-degree indices considered above are potential tools for QSPR analysis. 


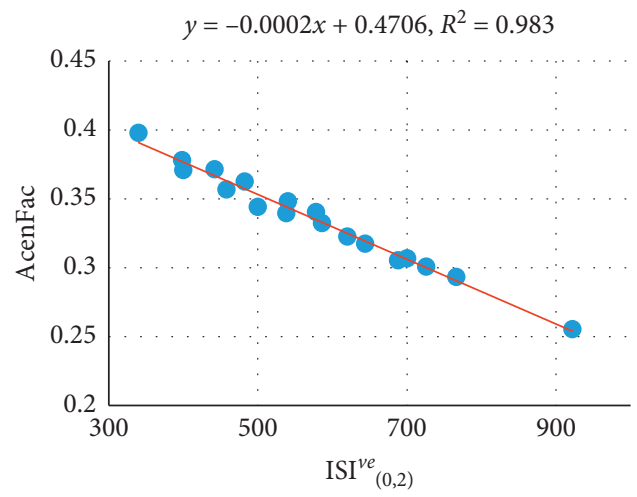

(a)

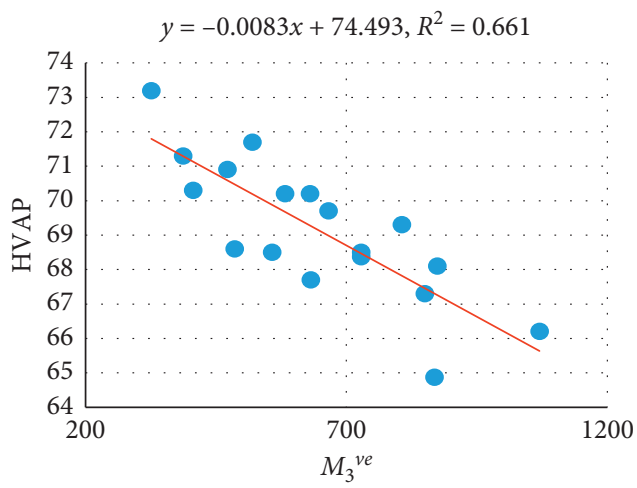

(c)

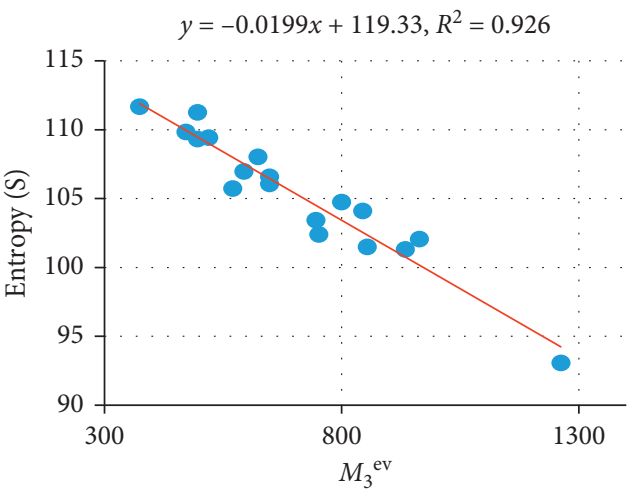

(b)

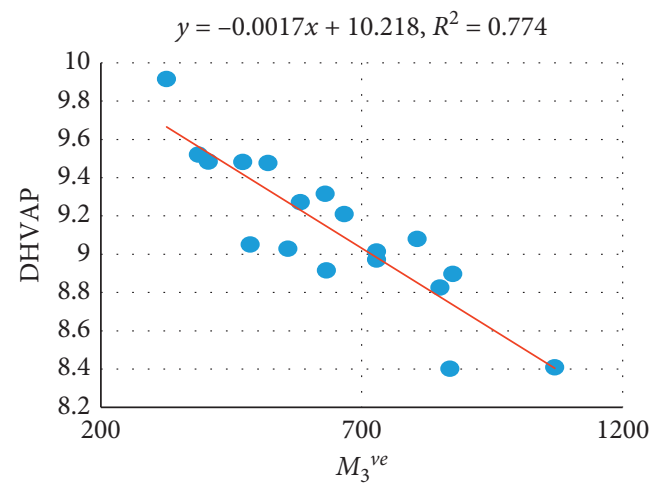

(d)

Figure 5: Correlation of $\operatorname{ISI}_{(0,2)}^{\mathrm{ve}}(G)$ with AcenFac, $M_{3}^{\text {ev }}(G)$ with entropy, and $M_{3}^{\mathrm{ve}}(G)$ with DHVAP and HVAP along with their equation of regression.

TABLE 13: The square of correlation between ve-degree topological indices and many physicochemical properties of octane isomers.

\begin{tabular}{lcccc}
\hline Index & Entropy $(S)$ & HVAP & DHVAP & AcenFac \\
\hline $\operatorname{ISI}_{(0,2)}^{v e}(G)=\mathrm{HM}_{1}^{v e}$ & 0.876795217 & 0.576521667 & 0.702923962 & $\mathbf{0 . 9 8 3 3 7 2 3 3 8}$ \\
$\operatorname{ISI}_{(2,0)}^{v e}(G)=\mathrm{HM}_{2}^{v e}$ & 0.869803837 & 0.548372117 & 0.679244658 & 0.969870048 \\
$M_{3}^{v e}(G)=F_{1}^{v e}$ & 0.858378682 & $\mathbf{0 . 6 6 1 1 8 4 5}$ & $\mathbf{0 . 7 7 4 4 9 0 4 7 7}$ & 0.979974381 \\
$M_{3}^{\text {ev }}(G)=F^{\text {ev }}$ & $\mathbf{0 . 9 2 6 2 0 7 5 3 1}$ & 0.632877013 & 0.751985132 & 0.963887056 \\
\hline
\end{tabular}

Bold values show highest values of correlation between topological indices and properties.

\section{Conclusion}

In this article, we have computed various ev-degree and vedegree topological indices of hyaluronic acid-curcumin/ paclitaxel conjugate using the general ve-inverse sum indeg index, general ve-Zagreb index, and general ev-degree index. The ve-degree index has been shown to have greater predictive ability and better correlation than classic degreebased indices; so the findings of the current studies will enable researchers to have a better understanding of the physicochemical and pharmacological characteristics of hyaluronic acid-curcumin/paclitaxel conjugates. Also, the predictive power of ve-degree indices have been tested on by using some physicochemical properties of octanes, and it is shown that these ve-degrees/ev-degree-based topological indices can be used as possible tools for QSPR. As these results are helpful in chemical science as well as pharmaceutical point of view, in this regard, the mathematical properties of hyaluronic acid-curcumin/paclitaxel conjugate are worth to investigate for future studies.

\section{Data Availability}

The data used to support the findings of this study are included within the article.

\section{Conflicts of Interest}

The authors declare that they have no conflicts of interest.

\section{Acknowledgments}

The authors gratefully acknowledge Qassim University, represented by the Deanship of Scientific Research, on the 
financial support for this research (10156-enuc-2020-1-3-I) (1.107-enuc-Y.Y.-I-Y-I) during the academic year 1441 $\mathrm{AH} / 2020 \mathrm{AD}$.

\section{References}

[1] J. C. Dearden, "The use of topological indices in QSAR and QSPR modeling," in Advances in QSAR Modeling, pp. 57-88, Springer, Berlin, Germany, 2017.

[2] A. Mauri, V. Consonni, and Roberto Todeschini, "Molecular descriptors," in Handbook of Computational Chemistry, J. Leszczynski, Ed., Springer Netherlands, Dordrecht, Netherlands, pp. 1-29, 2016.

[3] H. Wiener, "Structural determination of paraffin boiling points," Journal of the American Chemical Society, vol. 69, no. 1, pp. 17-20, 1947.

[4] I. Gutman, E. Milovanović, and I. Milovanović, "Beyond the Zagreb indices," AKCE International Journal of Graphs and Combinatorics, vol. 17, no. 1, pp. 74-85, 2018.

[5] A. Ali, L. Zhong, and I. Gutman, "Harmonic index and its generalizations: extremal results and bounds," Match, vol. 81, no. 2, pp. 249-311, 2019.

[6] K. C. Das, I. Gutman, and B. Furtula, "Survey on geometricarithmetic indices of graphs," MATCH Communications in Mathematical and in Computer Chemistry, vol. 65, no. 3, pp. 595-644, 2011.

[7] I. Gutman and N. Trinajstić, "Graph theory and molecular orbitals: total $\varphi$-electron energy of alternant hydrocarbons," Chemical Physics Letters, vol. 17, no. 4, pp. 535-538, 1972.

[8] I. Gutman and Ch. D. Kinkar, "The first Zagreb index 30 years after," MATCH Communications in Mathematical and in Computer Chemistry, vol. 50, no. 1, pp. 83-92, 2004.

[9] I. Gutman, B. Furtula, and V. Katanić, "Randić index and information," AKCE International Journal of Graphs and Combinatorics, vol. 15, no. 3, pp. 307-312, 2018.

[10] M. Randic, "Characterization of molecular branching," Journal of the American Chemical Society, vol. 97, no. 23, pp. 6609-6615, 1975.

[11] B. Zhou and N. Trinajstić, "On a novel connectivity index," Journal of Mathematical Chemistry, vol. 46, no. 4, pp. 12521270, 2009.

[12] B. Zhou and N. Trinajstić, "On general sum-connectivity index," Journal of Mathematical Chemistry, vol. 47, no. 1, pp. 210-218, 2010.

[13] M. Arockiaraj, J. Clement, and B. Krishnan, "Topological indices and their applications to circumcised donut benzenoid systems, kekulenes and drugs," Polycyclic Aromatic Compounds, vol. 40, no. 2, pp. 280-303, 2018.

[14] W. Gao, M. K. Siddiqui, M. Imran, M. Kamran Jamil, and M. Reza Farahani, "Forgotten topological index of chemical structure in drugs," Saudi Pharmaceutical Journal, vol. 24, no. 3, pp. 258-264, 2016.

[15] W. Gao, W. Wang, and M. Reza Farahani, "Topological indices study of molecular structure in anticancer drugs," Journal of Chemistry, vol. 2016, Article ID 3216327, 8 pages, 2016.

[16] W. Gao, Y. Wang, B. Basavanagoud, and M. Kamran Jamil, "Characteristics studies of molecular structures in drugs," Saudi Pharmaceutical Journal, vol. 25, no. 4, pp. 580-586, 2017.

[17] S. Mondal, N. De, and A. Pal, "Topological indices of some chemical structures applied for the treatment of COVID-19 patients," Polycyclic Aromatic Compounds, pp. 1-15, 2020.
[18] L. Zheng, Y. Wang, and W. Gao, "Topological indices of hyaluronic acid-paclitaxel conjugates' molecular structure in cancer treatment," Open Chemistry, vol. 17, no. 1, pp. 81-87, 2019.

[19] M. C. Shanmukha, N. S. Basavarajappa, K. C. Shilpa, and A. Usha, "Degree-based topological indices on anticancer drugs with QSPR analysis," Heliyon, vol. 6, no. 6, Article ID e04235, 2020.

[20] I. Sarkar, S. Goswami, and P. Majumder, "Quantitative structure-activity relationship (QSAR) study of some DNAintercalating anticancer drugs," in Computational Advancement in Communication Circuits and Systems, pp. 357-366, Springer, Berlin, Germany, 2020.

[21] Emmert-Streib and Frank, Statistical Modelling of Molecular Descriptors in QSAR/QSPR, John Wiley \& Sons, Hoboken, NJ, USA, 2012.

[22] S. A. K. Kirmani, P. Ali, and F. Azam, "Topological indices and QSPR/QSAR analysis of some antiviral drugs being investigated for the treatment of COVID-19 patients," International Journal of Quantum Chemistry, vol. 121, no. 9, Article ID e26594, 2021.

[23] M. Chellali, T. W. Haynes, S. T. Hedetniemi, and T. M. Lewis, "On ve-degrees and ev-degrees in graphs," Discrete Mathematics, vol. 340, no. 2, pp. 31-38, 2017.

[24] S. Ediz, "Predicting some physicochemical properties of octane isomers: a topological approach using ev-degree and ve-degree Zagreb indices," 2017, https://arxiv.org/abs/1701.02859.

[25] S. Ediz, "A new tool for QSPR researches: ev-degree Randić index," Celal Bayar Üniversitesi Fen Bilimleri Dergisi, vol. 13, no. 3, pp. 615-618, 2017.

[26] B. Sahin and S. Ediz, "On ev-degree and ve-degree topological indices," Iranian Journal of Mathematical Chemistry, vol. 9, no. 4, pp. 263-277, 2018.

[27] S. Ediz, "On ve-degree molecular topological properties of silicate and oxygen networks," International Journal of Computing Science and Mathematics, vol. 9, no. 1, pp. 1-12, 2018.

[28] B. Horoldagva, K. C. Das, and S. Tsend-Ayush, "On ve-degree and ev-degree of graphs," Discrete Optimization, vol. 31, pp. 1-7, 2019.

[29] V. R. Kulli, "On ve-degree indices and their polynomials of dominating oxide networks," Annals of Pure and Applied Mathematics, vol. 18, no. 1, pp. 1-7, 2018.

[30] V. R. Kulli, "Computing the F-ve-degree index and its polynomial of dominating oxide and regular triangulate oxide networks," International Journal of Fuzzy Mathematical Archive, vol. 16, no. 1s, pp. 1-6, 2018.

[31] V. R. Kulli, "On the square ve-degree index and its polynomial of certain oxide networks," Journal of Global Research in Mathematical Archives, vol. 5, no. 10, pp. 1-4, 2018.

[32] V. R. Kulli, "Two new arithmetic-geometric ve-degree indices," Annals of Pure and Applied Mathematics, vol. 17, no. 1, pp. 107-112, 2018.

[33] V. R. Kulli, "Computing ev-degree and multiplicative evdegree indices of certain chemical structures," International Journal of Engineering Sciences \& Research Technology, vol. 9, no. 7, pp. 54-65, 2020.

[34] S. Fajtlowicz, "On conjectures of graffiti-II," Congruent Number, vol. 60, pp. 187-197, 1987.

[35] L. B. Kier and L. H. Hall, "The meaning of molecular connectivity: a bimolecular accessibility model," Croatica Chemica Acta, vol. 75, no. 2, pp. 371-382, 2002.

[36] P. S. Ranjini, V. Lokesha, and A. Usha, "Relation between phenylene and hexagonal squeeze using harmonic index," 
International Journal of Graph Theory, vol. 1, no. 4, pp. 116-121, 2013.

[37] D. Vukičević and M. Gašperov, "Bond additive modeling 1. Adriatic indices," Croatica Chemica Acta, vol. 83, no. 3, pp. 243-260, 2010.

[38] M. Cancan, "On ev-degree and ve-degree topological properties of Tickysim spiking neural network," Computational Intelligence and Neuroscience, vol. 2019, Article ID 8429120, 7 pages, 2019.

[39] K. Yamaç and M. Cancan, "On ev-degree and ve-degree based topological properties of the sierpiński gasket fractal," Sigma, vol. 37, no. 4, pp. 1275-1280, 2019.

[40] M. Cancan, S. Ediz, M. Alaeiyan, and M. Reza Farahani, "On ve-degree molecular properties of copper oxide," Journal of Information and Optimization Sciences, vol. 41, no. 4, pp. 949-957, 2020.

[41] M. Cancan, S. Ediz, and M. Reza Farahani, "On ve-degree atom-bond connectivity, sum-connectivity, geometricarithmetic and harmonic indices of copper oxide," Eurasian Chemical Communications, vol. 2, no. 5, pp. 641-645, 2020.

[42] S.-B. Chen, Abdul Rauf, M. Ishtiaq, M. Naeem, and A. Aslam, "On ve-degree-and ev-degree-based topological properties of crystallographic structure of cuprite $\mathrm{Cu}_{2} \mathrm{O}$," Open Chemistry, vol. 19, no. 1, pp. 576-585, 2021.

[43] Z.-Q. Cai, Abdul Rauf, M. Ishtiaq, and M. K. Siddiqui, “On vedegree and ev-degree based topological properties of silicon carbide $\mathrm{Si}_{2} \mathrm{C}_{3}$-II $[p, Q]$," Polycyclic Aromatic Compounds, pp. 1-15, 2020.

[44] A. Rauf, M. Ishtiaq, M. K. Siddiqui, and R. Andleeb, "Topological properties of doxorubicin conjugated PEG-PAsp copolymer molecular structure used in cancer treatment," Polycyclic Aromatic Compounds, pp. 1-14, 2020.

[45] S. Ediz and M. Cancan, "Molecular topological properties of alkylating agents based anticancer drug candidates via some ve-degree topological indices," Current Computer-Aided Drug Design, vol. 16, no. 2, pp. 190-195, 2020.

[46] S. Ediz, M. Cancan, M. Alaeiyan, and M. Reza Farahani, "Vedegree and ev-degree topological analysis of some anticancer drugs," Eurasian Chemical Communications, vol. 2, no. 8, pp. 834-840, 2020.

[47] M. C. Wani, H. L. Taylor, M. E. Wall, P. Coggon, and A. T. McPhail, "Plant antitumor agents. VI. Isolation and structure of taxol, a novel antileukemic and antitumor agent from Taxus brevifolia," Journal of the American Chemical Society, vol. 93, no. 9, pp. 2325-2327, 1971.

[48] A. Giordano and G. Tommonaro, "Curcumin and cancer," Nutrients, vol. 11, no. 10, p. 2376, 2019.

[49] H. Batra, S. Pawar, and D. Bahl, "Curcumin in combination with anti-cancer drugs: a nanomedicine review," Pharmacological Research, vol. 139, pp. 91-105, 2019.

[50] M. Kharat and D. J. McClements, "Recent advances in colloidal delivery systems for nutraceuticals: a case study- delivery by design of curcumin," Journal of Colloid and Interface Science, vol. 557, pp. 506-518, 2019.

[51] I. E. Allijn, M. S. Raymond, and G. Storm, "Comparison of pharmaceutical nanoformulations for curcumin: enhancement of aqueous solubility and carrier retention," International Journal of Pharmaceutics, vol. 506, no. 1-2, pp. 407-413, 2016.

[52] S. Zhao, L. Ma, C. Cao, Q. Yu, L. Chen, and J. Liu, "Curcuminloaded redox response of self-assembled micelles for enhanced antitumor and anti-inflammation efficacy," International Journal of Nanomedicine, vol. 12, p. 2489, 2017.
[53] W.-T. Zhu, S.-Y. Liu, L. Wu et al., "Delivery of curcumin by directed self-assembled micelles enhances therapeutic treatment of non-small-cell lung cancer," International Journal of Nanomedicine, vol. 12, p. 2621, 2017.

[54] B. P. Toole, "Hyaluronan in morphogenesis," Seminars in Cell \& Developmental Biology, vol. 12, no. 2, pp. 79-87, 2001.

[55] H. Lee, H. Mok, S. Lee, Y.-K. Oh, and T. G. Park, "Targetspecific intracellular delivery of SiRNA using degradable hyaluronic acid nanogels," Journal of Controlled Release, vol. 119, no. 2, pp. 245-252, 2007.

[56] G. Tripodo, T. Adriana, M. Luisa Torre, G. Giammona, G. Trapani, and D. Mandracchia, "Hyaluronic acid and its derivatives in drug delivery and imaging: recent advances and challenges," European Journal of Pharmaceutics and Biopharmaceutics, vol. 97, pp. 400-416, 2015.

[57] G. Saravanakumar, V. G. Deepagan, R. Jayakumar, and J. H. Park, "Hyaluronic acid-based conjugates for tumortargeted drug delivery and imaging," Journal of Biomedical Nanotechnology, vol. 10, no. 1, pp. 17-31, 2014.

[58] K. Wang, C. Guo, D. Xue et al., "In vivo evaluation of reduction-responsive alendronate-hyaluronan-curcumin polymer-drug conjugates for targeted therapy of bone metastatic breast cancer," Molecular Pharmaceutics, vol. 15, no. 7, pp. 2764-2769, 2018.

[59] J. Buragohain, B. Deka, and A. Bharali, "A generalized ISI index of some chemical structures," Journal of Molecular Structure, vol. 1208, Article ID 127843, 2020.

[60] P. Ali, A. K. K. Syed, O. A. Rugaie, and F. Azam, "Degreebased topological indices and polynomials of hyaluronic acidcurcumin conjugates," Saudi Pharmaceutical Journal, vol. 28, no. 9, pp. 1093-1100, 2020. 